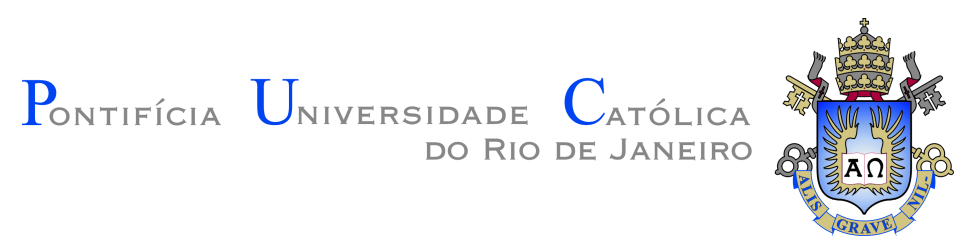

Hugo de Souza Oliveira

Modelo de mercado finito a tempo discreto

Dissertação apresentada como requisito parcial para obtenção do grau de Mestre pelo Programa de Pós-graduação em Matemática do Departamento de Matemática do Centro Técnico Científico da PUC-Rio.

Orientador :

Prof. Boyan Sirakov

Coorientador: Profa ${ }^{a}$ Ana Patrícia Carvalho Gonçalves 


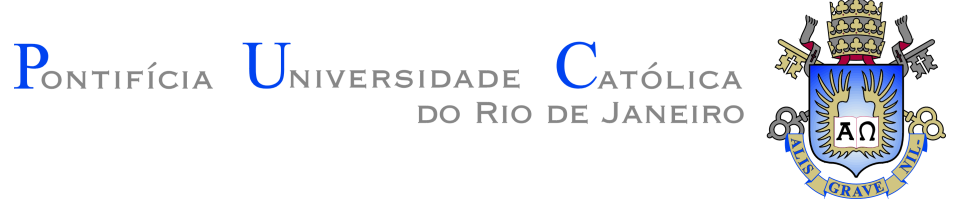

Hugo de Souza Oliveira

\title{
Modelo de mercado finito a tempo discreto
}

Dissertação apresentada como requisito parcial para obtenção do grau de Mestre pelo Programa de Pós-graduação em Matemática do Departamento de Matemática do Centro Técnico Científico da PUC-Rio. Aprovada pela Comissão Examinadora abaixo assinada.

\author{
Prof. Boyan Sirakov \\ Orientador \\ Departamento de Matemática - PUC-Rio
}

Prof ${ }^{a}$. Ana Patrícia Carvalho Gonçalves

Coorientador Instituto Superior Técnico - Universidade de Lisboa

Profa. Susan Schommer Universidade do Estado do Rio de Janeiro - UERJ

Prof. Davi Michel Valladão

Departamento de Engenharia Industrial - PUC-Rio

Prof. Simon Griffiths

Departamento de Matemática - PUC-Rio

Prof. Márcio da Silveira Carvalho

Coordenador Setorial do Centro Técnico Científico - PUC-Rio 
Todos os direitos reservados. É proibida a reprodução total ou parcial do trabalho sem autorização da universidade, do autor e do orientador.

\section{Hugo de Souza Oliveira}

Graduado em Ciências Econômicas pela Universidade Federal do Rio de Janeiro.

Ficha Catalográfica

de Souza Oliveira, Hugo

Modelo de mercado finito a tempo discreto / Hugo de Souza Oliveira; orientador: Boyan Sirakov; co-orientador: Ana Patrícia Carvalho Gonçalves. - 2017.

v., 67 f: il. color. ; $30 \mathrm{~cm}$

Dissertação (mestrado) - Pontifícia Universidade Católica do Rio de Janeiro, Departamento de Matemática.

Inclui bibliografia

1. Matemática - Teses. 2. Matemática Aplicada - Teses. 3. Precificação de Contratos;. 4. Martingal;. 5. Processos estocásticos;. 6. Arbitragem;. 7. Cobertura;. I. Sirakov, Boyan. II. Carvalho Gonçalves, Ana Patrícia. III. Pontifícia Universidade Católica do Rio de Janeiro. Departamento de Matemática. IV. Título. 
Dedico este trabalho à minha mãe, que me criou, me educou e sempre me apoiou. 


\section{Agradecimentos}

Agradeço à professora Ana Patrícia pela dedicação e orientação durante o mestrado, mesmo que à distância.

Agradeço ao professor Boyan por ter me aceitado como orientando, pela ajuda e pelo curso ministrado.

Agradeço também ao professor Simon pelas dicas, ajuda e aulas.

Agradeço e dedico à minha mãe e à Maria Cláudia, pelo apoio incondicional e carinho.

Por fim, agradeço à PUC-Rio, funcionários do Departamento de Matemática e professores que entenderam as dificuldades passadas. 


\section{Resumo}

de Souza Oliveira, Hugo; Sirakov, Boyan; Carvalho Gonçalves, Ana Patrícia. Modelo de mercado finito a tempo discreto. Rio de Janeiro, 2017. 67p. Dissertação de Mestrado - Departamento de Matemática, Pontifícia Universidade Católica do Rio de Janeiro.

O trabalho tem como objetivo ser uma introdução ao estudo de mercados financeiros tratados em tempo discreto com horizonte finito, bem como a dinâmica dos ativos financeiros principais. Descrevemos os tipos de ativos negociados em nosso mercado, dando enfoque aos contratos. Elaboraremos a hipótese central do modelo, a ausência de arbitragem e assim mostraremos como poderemos encontrar um preço correto ou então apresentaremos um intervalo de preços para os contratos. Posteriormente, mostraremos resultados gerais relativos à correta precificação de contratos, usando para isso os instrumentos de processos estocásticos e martingais. Apresentaremos alguns exemplos a título de ilustração.

\section{Palavras-chave}

Precificação de Contratos; Martingal; Processos estocásticos; Arbitragem; Cobertura; 


\section{Abstract}

de Souza Oliveira, Hugo; Sirakov, Boyan (Advisor); Carvalho Gonçalves, Ana Patrícia (Co-Advisor). Discrete time finite market model. Rio de Janeiro, 2017. 67p. Dissertação de Mestrado - Departamento de Matemática, Pontifícia Universidade Católica do Rio de Janeiro.

The dissertation aims to be an introduction to the study of financial markets in discrete time with finite horizon, as well as the dynamics of the main financial assets. We describe the types of assets traded in the market, focusing on contracts. We will elaborate the central hypothesis of the model, the absence of arbitrage and thus show how we can find a correct price or, at least, a range of prices of the contracts. Subsequently, we will show general results regarding how to find correct prices for contracts, using the machinery of stochastic processes and martingales.As an illustration, we present some examples.

\section{Keywords}

Pricing Contingent Claim; Martingale; Stochastic Processes; Arbitrage; Replicating Strategy; 


\section{Sumário}

1 Introdução $\quad 10$

2 Modelação matemática de um mercado financeiro $\quad 12$

2.1 Mercado 12

$\begin{array}{lll}2.2 \text { Ativos } & 12\end{array}$

2.3 Portfólios e Arbitragem 14

$\begin{array}{lll}2.4 & \text { A precificação de um contrato simples. } & 15\end{array}$

2.5 A Representação Matricial 20

2.6 A precificação de contratos em um mercado finito com dois períodos de tempo. 21

2.7 Um mercado com vários períodos de tempo. 27

3 Resultados Fundamentais $\quad 32$

3.1 Probabilidade Neutra ao Risco 32

$\begin{array}{ll}3.2 \text { Martingais } & 36\end{array}$

3.3 Integração Estocástica Discreta 39

3.4 O problema martingal 41

3.5 Teoremas Fundamentais 45

4 Modelo Binomial $\quad 53$

5 Conclusão $\quad 59$

$\begin{array}{ll}\text { Referências bibliográficas } & 60\end{array}$

$\begin{array}{ll}\text { A Probabilidade } & 61\end{array}$

A.1 Espaços Mensuráveis e Funções Mensuráveis 61

A.2 Processos Estocásticos 63

A.3 Independência e Esperança Condicional 64 
Funeral by funeral, theory advances.

Paul Samuelson 


\section{Introdução}

Modelar a dinâmica de ativos financeiros sempre foi algo desafiador devido a dificuldade de lidar com as incertezas dos preços em instantes futuros. Compreender como os preços flutuam pode ser uma tarefa de um investigador que busca uma explicação baseada em como os preços são formados, as leis de mercado e iterações entre agentes que transacionam os ativos.

Sob uma outra perspectiva, um investigador pode estudar o mercado e seus ativos supondo que estes têm comportamento puramente aleatório e assim utilizar a teoria da probabilidade a fim de obter respostas sobre os preços.

Dentre os muitos ativos financeiros que podem ser analisados sobre as diversas perspectivas encontram-se os contratos. Neste trabalho analisaremos sobre a perspectiva aleatória. Os contratos são ativos que assumem determinado valor em tempo futuro segundo os rumos que a natureza pode tomar, ou seja, tem seu valor atrelado a eventos aleatórios. Os contratos também podem ser funções que dependem do valor de outros ativos, estes também com preço variando segundo flutuações aleatórias.

O problema que este trabalho aborda é o de encontrar preços corretos de forma a que os contratos possam ser transacionados como um ativo com risco no mercado. O caso considerado é o de mercados com tempo finito, ou seja, há a delimitação de início e fim bem estabelecidos onde os ativos, incluindo os contratos, são negociados. Estes mercados são formados por finitos ativos com risco e livre de risco. Trataremos do caso em que há finitos estados da natureza, ou seja, há finitos eventos no espaço amostral e os ativos com risco podem assumir finitos valores em momentos futuros.

Para encontrarmos os preços corretos de contratos em mercados sob condições como as definidas no parágrafo anterior, usaremos ferramentas de processos estocásticos, em especial a dinâmica de informações será modelada usando filtros e martingais.

No segundo capítulo abordaremos os conceitos fundamentais do mercado. Desenvolveremos o conceito de arbitragem e mostraremos sua importância na precificação de contratos tanto num modelo de dois períodos de tempo quanto em um modelo de vários períodos de tempo. Os tipos de ativos serão definidos e a partir deles explicaremos como uma carteira é construída e tem seu valor 
definido. Também mostraremos quando é possível encontrar um preço correto de um contrato e quando o contrato tem um intervalo de preços corretos, ou seja, vários preços corretos. Também definiremos o conceito de mercados completos, onde os contratos sempre terão cobertura, e o conceito de mercados incompletos, onde os contratos nem sempre terão cobertura.

No terceiro capítulo introduziremos o conceito de probabilidade neutra ao risco que será usado extensivamente na sequência da dissertação. Mostraremos como esta medida artificial de probabilidade permite determinar o preço de ativos com risco quando tomamos o valor esperado, com respeito a esta medida, dos preços de tais ativos descontados pela taxa de juros.

Ainda no terceiro capítulo veremos os teoremas fundamentas desta dissertação. Os teoremas relacionam a existência de medidas de probabilidade neutra ao risco com a possibilidade de executar estratégias que resultem em arbitragem, bem como o fato do mercado em que estamos lidando ser completo ou não. Um breve exemplo do modelo binomial é apresentado no capítulo 4 para ilustrar a construção teórica desenvolvida. Por fim, apresentamos um apêndice com os principais resultados de probabilidade necessários para a leitura do texto. 


\section{Modelação matemática de um mercado financeiro}

Apresentaremos aqui o modelo de mercado usado neste trabalho de forma gradual. Definiremos o conceito de arbitragem, contratos, estratégias e com isso estudaremos como encontrar preços corretos para os contratos.

\section{1}

\section{Mercado}

Um mercado pode ser considerado como uma organização onde ofertantes e demandantes estabelecem uma relação comercial a fim de realizarem transações, acordos ou trocas comerciais. No caso aqui tratado, consideraremos o mercado onde agentes realizam trocas de ativos financeiros (especificamente ativos com risco, ativo sem risco e contratos, que mais à frente serão definidos) segundo suas necessidades e segundo preços tomados como informações dadas pelo próprio mercado.

O mercado será considerado em instantes discretos de tempo. A cada instante $n$ teremos as cotações dos preços dos ativos e em $n+1$ suas novas cotações, de forma que conseguiremos analisar sua variação e com isso utilizaremos estes dados em nosso modelo. Iniciaremos considerando somente dois períodos de tempo $(n=0$ e $n=1)$ e posteriormente trataremos de um mercado com $N$ períodos de tempo, onde $N \in \mathbb{N}$.

\section{2}

Ativos

Definiremos os ativos negociados em tal mercado. Podemos considerar a existência de um agente provedor de recursos financeiros que atua como um banco. Este agente é o responsável por disponibilizar o ativo livre de risco e cobrar juros sobre a variação do tempo. A taxa de juros cobrada sobre o ativo livre de risco é $r$ e é uma variável tomada como dada pelos agentes na economia. O banco atua como emprestador e depositário de dinheiro, pagando o retorno $B(1+r)$ em $n=1$ pela quantia $B$ que é depositada em $n=0$.

Consideraremos também uma quantidade finita $\ell \in \mathbb{N}$ de ativos com risco. Cada ativo com risco será notado por $S_{n}^{i} \operatorname{com} i \in\{1,2, \ldots, \ell\}$. O ativo com risco pode ser considerado uma ação ou qualquer outro produto financeiro 
cujo preço no período $n=1$ não é obtido de forma determinística, mas sim dependente dos estados da natureza. Em outras palavras, os preços no futuro são incertos, podendo haver alta, baixa ou estabilidade do preço, daí dizermos ser um ativo com risco.

Cabe aqui introduzirmos o que são os estados da natureza. Cada ativo de risco pode ser modelado como uma variável aleatória

$$
S^{i}: \Omega \longrightarrow \mathbb{R}
$$

tendo como domínio um conjunto $\Omega$. Em nosso modelo, trataremos apenas do caso em que $\Omega$ é um conjunto finito, ou seja, um conjunto com $k \in \mathbb{N}$ elementos, $\Omega=\left\{\omega_{1}, \omega_{2}, \ldots, \omega_{k}\right\}$. Cada $\omega_{j} \in \Omega$ é o que chamamos de um estado da natureza. Então para cada $\omega_{j}$ o mercado irá se comportar de uma maneira, pois todos os ativos com risco assumirão um valor respectivo a tal $\omega_{j}$. Para representar o mesmo ativo com risco em diferentes datas de tempo usaremos o subscrito $n$. Desta forma, $S_{0}^{i}$ representa o preço do ativo $S^{i}$ no tempo $n=0$. Em $n=0$ o preço é conhecido por todos no mercado, $S_{0}^{i}$ é uma constante. Agora, o preço em $n=1$ apresenta um comportamento aleatório, ou seja, $S_{1}^{i}$ é uma variável aleatória que pode assumir finitos valores.

Cabe aqui definir o que são os retornos de um ativo. Claro que o retorno dependerá do estado futuro do ativo em questão.

Definição 2.1 Cada ativo tem uma taxa de retorno $a_{i}$ definida como

$$
a_{i}=\frac{S_{1}^{i}-S_{0}^{i}}{S_{0}^{i}}
$$

que também depende de $\omega_{j}$, pois $S_{1}^{i}$ depende de $\omega_{j}$.

Agora definiremos o ativo que será estudado no trabalho, o contrato. Um contrato é um ativo que quando adquirido promete pagar a quem o possui uma determinada quantia que depende do estado futuro do mercado. Em outras palavras, um contrato é uma variável aleatória positiva.

Definição 2.2 Um contrato é uma variável aleatória $F: \Omega \rightarrow \mathbb{R}$, com $F\left(\omega_{j}\right) \geqslant 0 \forall \omega_{j} \in \Omega$.

Um contrato pode ser utilizado como ferramenta de proteção contra possíveis infortúnios, como também sendo parte de uma estratégia que visa realizar lucros em $n=1$. Como exemplo de contrato podemos citar os contratos futuros, que são muito usados como instrumentos de proteção, e as opções, que podem ser de diferentes tipos e permitem uma grande quantidade de estratégias. A princípio trataremos de contratos de maneira geral e posteriormente trataremos de tipos de contratos específicos. 


\section{3}

\section{Portfólios e Arbitragem}

O mercado constituído por 1 ativo livre de risco e $\ell$ ativos de risco pode ser representado por um vetor aleatório $M_{n}: \Omega \longrightarrow \mathbb{R}^{\ell+1}, n \in\{0,1\}$, onde para cada $j \in\{1,2, \ldots, k\}$ se tem

$$
M_{n}\left(\omega_{j}\right)=\left(B(1+r)^{n}, S_{n}^{1}\left(\omega_{j}\right), S_{n}^{2}\left(\omega_{j}\right), \ldots, S_{n}^{\ell}\left(\omega_{j}\right)\right)
$$

Em $n=0$ os preços dos ativos são determinísticos.

O número de ativos de um agente é chamado de portfólio ou estratégia e pode ser representado por um vetor.

Definição 2.3 Uma estratégia consiste em um vetor $\Gamma$ de $\mathbb{R}^{\ell+1}$ variáveis que indica o número de ativos que o agente possui. A i-ésima coordenada de tal vetor representa a posição em relação ao i-ésimo ativo. Desta forma se

$$
\Gamma=\left(\beta, \alpha_{1}, \alpha_{2}, \ldots, \alpha_{\ell}\right)
$$

é a estratégia de um agente e se $\alpha_{i}>0$ o agente possui a quantidade $\alpha_{i}$ do respectivo ativo $S^{i}$, enquanto que se $\alpha_{i}<0$ o agente deve a quantidade $\alpha_{i}$ do respectivo ativo. Se $\beta>0$ então o agente possui $\beta$ unidades no ativo livre de risco e se $\beta<0$ este mesmo agente deve a quantidade $\beta$ do ativo livre de risco.

Sabendo como representar o mercado a cada instante $n$ e sabendo a representação do portfólio, vamos definir o valor do portfólio perante aquele mercado.

Definição 2.4 O valor de um portfólio $\Gamma$ é definido como

$$
V_{n}^{\Gamma}\left(\omega_{j}\right):=\Gamma \cdot M_{n}\left(\omega_{j}\right)
$$

onde. é o produto escalar entre o vetor portfólio $\Gamma$ e o vetor aleatório $M_{n}$ que representa o mercado. O produto escalar é bem definido, pois ambos os vetores tem $\ell+1$ coordenadas.

No modelo de mercado que desenvolvemos estamos interessados em analisar uma característica específica, chamada de arbitragem, que nos irá auxiliar a encontrar preços corretos para contratos. Para tal, o mercado deve ser considerado livre de arbitragem, pois a arbitragem é a possibilidade de um agente realizar lucro sem haver risco de perda.

Definição 2.5 Um portfólio $\Gamma$ é uma oportunidade de arbitragem se 

1. $V_{0}^{\Gamma}=0$;
2. $V_{1}^{\Gamma}\left(\omega_{j}\right) \geqslant 0$ para todo $\omega_{j} \in \Omega$;
3. $V_{1}^{\Gamma}\left(\omega_{j}\right)>0$ para algum $\omega_{j} \in \Omega$.

Segundo a Definição 2.5, uma estratégia $\Gamma$ é uma estratégia de arbitragem quando é possível que alguém a assuma em $n=0$ sem despender quantia alguma e tenha probabilidade positiva de realizar lucro em $n=0$, sem o risco de perda.

Outra forma de definir a existência de arbitragem é notar que estamos arbitrando em um mercado se $V_{0}^{\Gamma}<0$ e $V_{1}^{\Gamma} \geqslant 0$ para todo $\omega_{j}$ em $\Omega$, com $V_{1}^{\Gamma}>0$ para algum $\omega_{j} \in \Omega$. Essas duas desigualdades dizem que em $n=0$ o agente tinha um valor negativo com a estratégia $\Gamma$ e independentemente do cenário em $n=1$, consegue fechar sua posição devedora sem risco algum. Podemos mostrar que de fato é uma arbitragem segundo a Definição 2.5. Notemos que se em $n=0$ acrescentarmos uma quantia $\theta B_{0}=\gamma>0$ ao valor do portfólio $\Gamma, B_{0}$ o valor do ativo livre de risco em $n=0$, de forma que

$$
V_{0}^{\Gamma}+\gamma=0
$$

podemos dizer que na verdade construímos o portfólio $\Gamma^{\prime}=\left(\beta+\theta, \alpha_{1}, \ldots \alpha_{\ell}\right)$. Em $n=1$ ocorrerá que

$$
\begin{aligned}
& V_{1}^{\Gamma^{\prime}}=(\beta+\theta) B_{1}+\ldots+\alpha_{n} S_{1}^{\ell} \\
& V_{1}^{\Gamma^{\prime}}=V_{1}^{\Gamma}+\theta B_{1}>0 .
\end{aligned}
$$

Em outras palavras, se algum agente consegue construir um portfólio que de $n=0$ para $n=1$ passe de um valor negativo a um valor positivo, com probabilidade positiva, então também está realizando arbitragem.

\section{4}

\section{A precificação de um contrato simples.}

O problema central deste trabalho é o de precificar corretamente um contrato e precisar sob que condições isso é possível. Qual seria o preço correto hoje de um instrumento financeiro que dá a possibilidade de realizar lucro em uma data futura e de que variáveis esse preço dependeria?

Consideremos um caso simples. Suponhamos que temos um mercado com somente dois ativos, $B$ representando o ativo sem risco e $S$ representando o 
ativo com risco. O preço do ativo sem risco será 1 unidade monetária e do ativo com risco será $s$ unidades monetárias. Este mercado está definido para dois períodos de tempo, $n=0$ e $n=1$, e dois estados da natureza, ou seja, o espaço amostral é $\Omega=\left\{\omega_{1}, \omega_{2}\right\}$. Consideremos que os possíveis retornos do ativo de risco são $a$ e $b$.

Teorema 2.6 (Ausência de arbitragem) Um mercado muito simples como o definido acima é livre de arbitragem se, e somente se, $a<r<b$.

Prova. A prova é feita por redução ao absurdo. O preço em $n=0$ do ativo com risco é denotado por $s$. Denotaremos a taxa de juros do mercado por $r$. Também consideremos o espaço amostral $\Omega=\left\{\omega_{1}, \omega_{2}\right\}$. Suponha que $a \geqslant r$. A taxa de retorno $a$, é igual ou mais atraente do que a taxa de juros $r$ do mercado. Então um agente deve considerar a compra do ativo de risco ao invés do ativo livre de risco. Suponha que o agente peça a quantia $s$ emprestada ao banco, pagando a taxa de juros $r$ e adquira uma unidade do ativo de risco. Seu portfólio será então representado por $\Gamma=(-s, 1)$. Em $n=1$ os preços ficam $(1+r)$ para o ativo livre de risco e $S_{1}\left(\omega_{1}\right)=s(1+a)$ ou $S_{1}\left(\omega_{2}\right)=s(1+b)$ para o ativo com risco. Para todo caso temos que $s(1+b)>s(1+a) \geqslant s(1+r)$.

Vemos que

$$
\begin{aligned}
V_{1}^{\Gamma}\left(\omega_{1}\right) & =(-s, 1) \cdot M_{1}\left(\omega_{1}\right) \\
& =-s(1+r)+S_{1}\left(\omega_{1}\right) \\
& =-s(1+r)+s(1+a)
\end{aligned}
$$

$\mathrm{Ou}$

$$
\begin{aligned}
V_{1}^{\Gamma}\left(\omega_{2}\right) & =(-s, 1) \cdot M_{1}\left(\omega_{2}\right) \\
& =-s(1+r)+S_{1}\left(\omega_{2}\right) \\
& =-s(1+r)+s(1+b) .
\end{aligned}
$$

Pela desigualdade entre os retornos é claro que $V_{1}^{\Gamma}\left(\omega_{2}\right)>V_{1}^{\Gamma}\left(\omega_{1}\right) \geqslant 0$. Dessa forma, a partir de $V_{0}^{\Gamma}=0$ é possível obter $V_{1}^{\Gamma} \geqslant 0$, temos arbitragem.

Suponha agora que $r \geqslant b$. Torna-se atraente adquirir o ativo livre de risco $B$. Um agente pode tomar emprestado o ativo de risco e vendê-lo, ficando com a quantia $s$ (que é o preço em $n=0$ do ativo com risco) livre de risco e portanto a estratégia agora é representada por: $\Gamma=(s,-1)$. Agora teremos no mercado $s(1+r) \geqslant s(1+b)>s(1+a)$. O agente deve retornar o ativo que tomou 
emprestado. Logo, em $n=1$ este agente estará com o valor do portfólio

$$
\begin{aligned}
V_{1}^{\Gamma}\left(\omega_{1}\right) & =(s,-1) \cdot M_{1}\left(\omega_{1}\right) \\
& =s(1+r)-S_{1}\left(\omega_{1}\right) \\
& =s(1+r)-s(1+a)
\end{aligned}
$$

$\mathrm{Ou}$

$$
\begin{aligned}
V_{1}^{\Gamma}\left(\omega_{2}\right) & =(s,-1) \cdot M_{1}\left(\omega_{2}\right) \\
& =s(1+r)-S_{1}\left(\omega_{2}\right) \\
& =s(1+r)-s(1+b) .
\end{aligned}
$$

Portanto, temos $V_{2}^{\Gamma}\left(\omega_{1}\right)>V_{1}^{\Gamma}\left(\omega_{2}\right) \geqslant 0$. Novamente há uma oportunidade de arbitragem.

Note que quando $r \leqslant a$ ou $r \geqslant b$ há arbitragem. Vamos mostrar a outra implicação, ou seja, se $a<r<b$ então não há oportunidades de arbitragem. Tome um portfólio $\Gamma=(\beta, \alpha)$ tal que $V_{0}^{\Gamma}=0$, ou seja, construído de forma que as posições do agente em ativo livre de risco e ativo com risco se compensem e resultem em um valor nulo. Para que ocorra a igualdade $V_{0}^{\Gamma}=0$, deveremos ter $s \alpha=-\beta$, pois $V_{0}^{\Gamma}=(\beta, \alpha)$. $(1, s)$. Considere $\alpha \neq 0$, caso contrário nunca há arbitragem, pois o portfólio seria o vetor nulo. Usando o portfólio $\Gamma=(-s \alpha, \alpha)$, teremos em $n=1$ :

$$
\begin{gathered}
V_{1}^{\Gamma}\left(\omega_{1}\right)=-s \alpha(1+r)+\alpha S_{1}\left(\omega_{1}\right)=-s \alpha-s \alpha r+\alpha s+\alpha s a=\alpha s(a-r), \\
V_{1}^{\Gamma}\left(\omega_{2}\right)=-s \alpha(1+r)+\alpha S_{1}\left(\omega_{2}\right)=-s \alpha+-s \alpha r+\alpha s+\alpha s b=\alpha s(b-r) .
\end{gathered}
$$

Como tomamos por hipótese $a<r<b$, temos $V_{1}^{\Gamma}\left(\omega_{1}\right)<0<V_{1}^{\Gamma}\left(\omega_{2}\right)$ e com isso não temos $V_{1}^{\Gamma} \geqslant 0$ para todo $\omega_{i} \in \Omega$, portanto, não há arbitragem.

Agora vamos nos dedicar ao tema principal da dissertação. Neste ponto trataremos de um contrato em um ambiente simples. Usando o conceito de arbitragem e fatos que mostramos ainda à pouco, mostraremos como a ausência de arbitragem em um mercado é uma característica desejável e como permite a correta precificação de um contrato.

Note que ao longo do trabalho estaremos desconsiderando totalmente os custos de transação. Esta hipótese é irreal, porém útil para facilitar uma primeira abordagem ao estudo dos mercados e precificação de contratos.

Em $n=0$ queremos determinar o preço $P$ do contrato. Consideremos um mercado sem arbitragem igual ao definido acima: 


\begin{tabular}{c|cc} 
Preço em $\mathrm{n}=0$ & \multicolumn{2}{|c}{ Preço em $\mathrm{n}=1$} \\
\hline 1 & $(1+r)$ & $(1+r)$ \\
$s$ & $s(1+a)$ & $s(1+b)$
\end{tabular}

Tabela 2.1: Exemplo de mercado

com $a<r<b$, onde $a$ e $b$ são as possíveis taxas de retorno do ativo com risco, e uma estratégia $\Gamma=(\beta, \alpha)$. Estamos considerando $B=1$, ou seja, o ativo livre de risco tem preço unitário em $n=0$. Podemos supor que $\Gamma$ é tal que

$$
V_{0}^{\Gamma}=\Gamma \cdot M_{0}=P
$$

onde $P$ é o valor do portfólio em $n=0$.

$$
V_{1}^{\Gamma}=\Gamma \cdot M_{1}\left(\omega_{i}\right)=F\left(\omega_{i}\right)
$$

e $F\left(\omega_{1}\right)=f_{1}, F\left(\omega_{2}\right)=f_{2}$.

Desta forma, $(\beta, \alpha) \cdot(1, s)=\beta+\alpha s=P$.

$$
\begin{aligned}
& \beta(1+r)+\alpha S_{1}\left(\omega_{1}\right)=f_{1} \\
& \beta(1+r)+\alpha S_{1}\left(\omega_{2}\right)=f_{2}
\end{aligned}
$$

Agora fazemos (2-3) - (2-2) e (2-2) + (2-3) para obter as seguintes equações:

$$
\begin{gathered}
\alpha s=\frac{f_{2}-f_{1}}{(b-a)} \\
\beta=\frac{f_{2}+f_{1}-\alpha s(a+b+2)}{2(1+r)}
\end{gathered}
$$

Substituímos (2-4) em (2-5) e obtemos:

$$
\beta=\frac{f_{2}+f_{1}+\left(\frac{f_{1}-f_{2}}{b-a}\right)(a+b+2)}{2(1+r)},
$$

e após simplificar:

$$
\beta=\frac{f_{1}(1+b)-f_{2}(1+a)}{(1+r)(b-a)}
$$

Então nosso portfólio é:

$$
\Gamma=\left(\frac{f_{1}(1+b)-f_{2}(1+a)}{(1+r)(b-a)}, \frac{f_{2}-f_{1}}{(b-a) s}\right)
$$


Como $\beta+s \alpha=P$, realizamos esta conta com $\alpha$ e $\beta$ que acabamos de encontrar para acharmos o preço $P$ do contrato:

$$
P=\frac{f_{2}(r-a)+f_{1}(b-r)}{(1+r)(b-a)} .
$$

Daí vemos que o preço do contrato depende somente de variáveis que não são aleatórias. O preço correto só depende das informações do mercado e não de cada estado da natureza $\omega_{i} \in \Omega$.

O preço correto do contrato em um mercado sem arbitragem, como este que estamos considerando, deve ser único.

Suponha que um agente A compre o contrato de um agente B pagando um preço $P^{\prime}>V_{0}^{\Gamma}=P$. O agente $\mathrm{B}$ adquire o portfólio $\Gamma$ em $n=0$. Em $n=1$ o agente B pagará ao agente $\mathrm{A}$ a quantia obrigatória firmada em contrato que representamos por $F(\omega)$. Como para pagar esta quantia B deverá ter liquidado $V_{1}^{\Gamma}$, vemos que ele terá lucro de $P^{\prime}-P>0$, logo há arbitragem.

Agora suponha que A pague $P^{\prime \prime}<P$ a B pelo contrato. Para isso, A toma $V_{0}^{\Gamma}$ por empréstimo em $n=0$ e compra $P^{\prime \prime}$. Em $n=1$, A recebe $F$ graças ao contrato e paga o que devia ao banco pelo empréstimo. $\mathrm{O}$ agente $\mathrm{A}$ tem lucro de $P-P^{\prime \prime}$ nessa operação, usando somente arbitragem.

No modelo simples de mercado, é possível ver que o contrato possui um preço correto exato. Mas isso nem sempre ocorre. Um contrato pode ter mais do que um preço correto e isso depende das características do mercado em que está sendo negociado.

Como já descrito anteriormente, o contrato $F$ é uma variável aleatória $F: \Omega \longrightarrow \mathbb{R}$ com $\Omega=\left\{\omega_{1}, \omega_{2}, \ldots \omega_{k}\right\} ; k \in \mathbb{N}$.

A partir de agora denotamos o preço correto do contrato por $P_{0}(F)$.

Há duas posições que um agente pode assumir em um contrato: a posição larga e a posição curta. Na posição larga o agente receberá a quantia $F\left(\omega_{j}\right)$ no tempo final $(n=1)$ e na posição curta ele pagará a quantia $F\left(\omega_{j}\right)$ em $n=1$. Em $n=0$ os agentes tomam suas respectivas posições comprando e vendendo o contrato em quantidades $\theta \in \mathbb{R}$ pelo preço $P_{0}(F)$. Para definir o preço $P_{0}(F)$ de forma correta é necessário apresentar algumas definições.

Definição 2.7 Um contrato $F: \Omega \longrightarrow \mathbb{R}$ possui cobertura em um mercado $M_{n}$ se existe um portfólio ou estratégia $\Gamma \in \mathbb{R}^{\ell+1}$ tal que $V_{1}^{\Gamma}(\omega)=F(\omega), \forall \omega \in \Omega$.

Notemos que o preço correto do contrato, no exemplo acima, foi definido utilizando um portfólio que replica o valor do contrato. Ou seja, $\Gamma$ é uma cobertura do contrato e, para que não ocorra arbitragem, $V_{0}^{\Gamma}=P_{0}(F)$.

Um contrato pode possuir mais de uma cobertura assim como nenhuma cobertura. 
Definição 2.8 Um mercado $M_{n}$ livre de arbitragem é dito completo quando para todo contrato existe uma cobertura. Se há algum contrato sem cobertura então o mercado é dito incompleto.

\section{5}

\section{A Representação Matricial}

As definições de mercado, contrato e portfólio podem ser apresentadas em notação matricial. Contratos e ativos de risco tem seus valores em $n=1$ definidos segundo os estados da natureza $\Omega=\left\{\omega_{1}, \omega_{2}, \ldots \omega_{k}\right\}$.

Dessa forma, a cada contrato $F$ podemos definir o vetor com todos os resultados possíveis deste contrato:

$$
\left(F\left(\omega_{1}\right), F\left(\omega_{2}\right), \ldots, F\left(\omega_{k}\right)\right) \in \mathbb{R}^{k}
$$

O mercado assumirá vários estados futuros segundo os estados da natureza $\Omega$. Desta forma, podemos representá-lo por uma matriz $\mathbb{S}_{(\ell+1) \times k}$, onde

$$
\mathbb{S}=\left(\begin{array}{cccc}
1+r & 1+r & \ldots & 1+r \\
S_{1}^{1}\left(\omega_{1}\right) & S_{1}^{1}\left(\omega_{2}\right) & \ldots & S_{1}^{1}\left(\omega_{k}\right) \\
\vdots & \vdots & \ddots & \vdots \\
S_{1}^{\ell}\left(\omega_{1}\right) & S_{1}^{\ell}\left(\omega_{2}\right) & \ldots & S_{1}^{\ell}\left(\omega_{k}\right)
\end{array}\right)
$$

onde cada linha $i$ indica o preço de cada ativo $i$ em $n=1$ e cada coluna $j$ indica o estado da natureza que está sendo considerado. Especificamente, $i \in\{0,1, \ldots \ell\}$ e $j \in\{1,2, \ldots k\} . \mathbb{S}^{T}$, a transposta de $\mathbb{S}$, é uma transformação linear tal que;

$$
\mathbb{S}^{T}: \mathbb{R}^{\ell+1} \longrightarrow \mathbb{R}^{k}
$$

Aplicando a transformação linear $\mathbb{S}^{T}$ acima a um portfólio $\Gamma=$ $\left(\beta, \alpha_{1}, \alpha_{2}, \ldots \alpha_{\ell}\right)$ teremos como resultado um vetor em $\mathbb{R}^{k}$ onde cada coordenada corresponde ao valor de $\Gamma$ sob um estado diferente da natureza. $\mathrm{Ou}$ seja,

$\mathbb{S}^{T}(\Gamma)=\left(\begin{array}{cccc}1+r & S_{1}^{1}\left(\omega_{1}\right) & \ldots & S_{1}^{\ell}\left(\omega_{1}\right) \\ 1+r & S_{1}^{1}\left(\omega_{2}\right) & \ldots & S_{1}^{\ell}\left(\omega_{2}\right) \\ \vdots & \vdots & \ddots & \vdots \\ 1+r & S_{1}^{1}\left(\omega_{k}\right) & \ldots & S_{1}^{\ell}\left(\omega_{k}\right)\end{array}\right) \cdot\left(\begin{array}{c}\beta \\ \alpha_{1} \\ \vdots \\ \alpha_{\ell}\end{array}\right)=\left(V_{1}^{\Gamma}\left(\omega_{1}\right), V_{1}^{\Gamma}\left(\omega_{2}\right), \ldots, V_{1}^{\Gamma}\left(\omega_{k}\right)\right)$

Temos que $F \in \operatorname{Im}\left(\mathbb{S}^{T}\right)$ se e somente se, existe $\Gamma \in \mathbb{R}^{\ell+1}$ tal que $V_{1}^{\Gamma}=F$. Logo o contrato possui cobertura se e somente se está na imagem da aplicação 
linear $\mathbb{S}^{T}$. Podemos verificar mais ainda. Se a aplicação é sobrejetiva então todo contrato possui cobertura. Logo o mercado é completo se e somente se $\mathbb{S}^{T}$ é sobrejetiva.

Pelo Teorema do Núcleo e Imagem sabemos que $\operatorname{dim} \operatorname{Ker}\left(\mathbb{S}^{T}\right)+\operatorname{dim} \operatorname{Im}\left(\mathbb{S}^{T}\right)=$ $\operatorname{dim} \mathbb{R}^{\ell+1}, \operatorname{logo} \operatorname{dim} \operatorname{Ker}(\mathbb{S})+\operatorname{dim} \operatorname{Im}\left(\mathbb{S}^{T}\right)=\ell+1$. Se o mercado é completo então $\mathbb{S}^{T}$ é sobrejetiva e temos

$$
\begin{array}{r}
\operatorname{dimKer}\left(\mathbb{S}^{T}\right)+k=\ell+1 \\
k \leqslant \ell+1
\end{array}
$$

Portanto, se $k>\ell+1$ então $M_{n}$ nunca será completo.

\section{6}

A precificação de contratos em um mercado finito com dois períodos de tempo.

Nesta seção mostraremos de forma rigorosa o que é o preço correto de um contrato e como o contrato torna-se um ativo quando negociado com este mesmo preço correto.

Um agente pode possuir um contrato e negociá-lo no mercado como um novo ativo de risco. O contrato pode ser transacionado em quantidades $\theta \in \mathbb{R}$ pelo preço $P_{0}(F)$, ou seja, um agente pode comprar ou vender unidades fracionadas $\theta$ do contrato.

Definição 2.9 Seja $M_{n}$ um mercado sem arbitragem e $F$ um contrato cujo preço é $P_{0}(F)$. Então o prȩ̧o correto de $F$ em $M_{n}$ é $P_{0}(F)$ se o novo mercado obtido agregando o contrato como um ativo com risco, $M_{n}^{\prime}:=$ $\left(B_{n}, S_{n}^{1}, \ldots S_{n}^{\ell}, F_{n}\right)$ com $F_{0}=P_{0}(F)$ e $F_{1}=F$ é livre de arbitragem.

Agora será provado, para o caso em que o mercado $M_{n}$ possui $\ell+1$ ativos e $M_{n}$ é livre de arbitragem, que um contrato $F$ que possui cobertura $\Gamma$ tem um único preço correto.

Proposição 2.10 Seja $M_{n}$ um mercado livre de arbitragem e F um contrato. Se F possui cobertura $\Gamma$ então seu único preço correto é $P_{0}(F)=V_{0}^{\Gamma}$.

A prova desta proposição mostrará que para casos em que o preço designado ao contrato seja diferente do que é considerado o preço justo, então haverá oportunidades de arbitragem em $M_{n}$. O nome preço justo é dado exatamente por ser este o preço que evita a existência de arbitragem em $M_{n}$.

Prova. Seja $\Gamma$ a cobertura do contrato $F$. Logo $V_{1}^{\Gamma}\left(\omega_{j}\right)=F\left(\omega_{j}\right) \quad \forall \omega_{j} \in \Omega$. Suponhamos que o contrato $F$ esteja sendo precificado no mercado por $P$, tal que $P>V_{0}^{\Gamma}$. 
Um agente perspicaz notará que o contrato está sendo cotado por um preço acima do correto. Dado isso, seria inteligente vender tal contrato por $P$ e, com o rendimento obtido, adquirir o portfólio de cobertura deste contrato, embolsando a diferença. Considerando o mercado $M_{n}^{\prime}=\left(B_{n}, S_{n}^{1}, \ldots, S_{n}^{\ell}, F_{n}\right)$ com o contrato sendo mais um ativo negociado, a estratégia descrita anteriormente seria denotada por $\Gamma^{\prime}=\left(\beta, \alpha_{1}, \ldots, \alpha_{\ell},-1\right)$, tal que o portfólio de cobertura é $\Gamma^{\prime}=\left(\beta, \alpha_{1}, \ldots, \alpha_{\ell}\right)$. Assim

$$
\begin{aligned}
V_{0}^{\Gamma^{\prime}} & =\Gamma^{\prime} \cdot M_{0}^{\prime} \\
& =\left(\beta, \alpha_{1}, \ldots, \alpha_{\ell},-1\right) \cdot\left(B_{0}, S_{0}^{1}, \ldots, S_{0}^{\ell}, P\right) \\
& =\left(\beta, \alpha_{1}, \ldots, \alpha_{\ell}\right) \cdot\left(B_{0}, S_{0}^{1}, \ldots, S_{0}^{\ell}\right)-P \\
& =\Gamma \cdot M_{0}-P \\
& =V_{0}^{\Gamma}-P<0
\end{aligned}
$$

A desigualdade $V_{0}^{\Gamma}-P<0$ indica que após adquirir $\Gamma^{\prime}$ ainda sobrou dinheiro em $n=0$, que pode ser investido em uma conta bancária e render à taxa $r$ ao agente.

Em $n=1$ ocorrerá que $V_{1}^{\Gamma}=F$ e o agente cumprirá suas obrigações pagando o que exige o contrato que vendeu. Agora vemos que houve lucro de pelo menos $P-V_{0}^{\Gamma}$ sem que se assumisse risco, então ocorreu uma oportunidade de arbitragem, logo $P$ não é o preço correto.

Suponhamos agora que $P<V_{0}^{\Gamma}$. Nesta situação, o contrato está abaixo do preço correto, logo é atraente comprá-lo. O agente pode tomar o portfólio $\Gamma$ emprestado, vender e adquirir o contrato. Para isso, tomemos a estratégia indicada pelo portfólio $\Gamma^{\prime \prime}=\left(-\beta,-\alpha^{1}, \ldots-\alpha^{\ell}, 1\right)$, ou seja, vende-se o portfólio $\Gamma$ e adquire o contrato ao preço $P$. Então

$$
\begin{aligned}
V_{0}^{\Gamma^{\prime \prime}} & =\Gamma^{\prime \prime} \cdot M_{0}^{\prime} \\
& =\left(-\beta,-\alpha^{1}, \ldots-\alpha^{\ell}, 1\right) \cdot\left(B_{0}, S_{0}^{1}, \ldots, S_{0}^{\ell}, P\right) \\
& =\left(-\beta,-\alpha^{1}, \ldots-\alpha^{\ell}\right) \cdot\left(B_{0}, S_{0}^{1}, \ldots, S_{0}^{\ell}\right)+P \\
& =-\Gamma \cdot M_{0}+P \\
& =-V_{0}^{\Gamma}+P<0
\end{aligned}
$$

Em $n=1$ teremos $V_{1}^{\Gamma}=F$, logo o agente recebe a quantia $V_{1}^{\Gamma}$ e fecha sua posição em relação ao que devia por ter adquirido o portfólio $\Gamma$. Em $n=0$ o agente havia realizado lucro, que poderia ter aplicado no ativo sem risco a taxa $r$. Então, há oportunidade de arbitragem em $M_{n}^{\prime}$, logo $P$ não é o preço correto. 
Mostramos que quando o preço difere de $V_{0}^{\Gamma}$ há oportunidades de arbitragem, e agora devemos mostrar que isso não ocorre quando há a igualdade. Suponha que o contrato seja precificado por $P=V_{0}^{\Gamma}$.

Consideremos $\Gamma^{\prime}$ uma oportunidade de arbitragem no mercado $M_{n}^{\prime}$. Temos que $\Gamma^{\prime}$ pode ser da forma $\Gamma^{\prime}=\left(\bar{\beta}, \bar{\alpha}^{1}, \ldots, \bar{\alpha}^{\ell}, 1\right)$ ou então $\Gamma^{\prime}=$ $\left(\bar{\beta}, \bar{\alpha}^{1}, \ldots, \bar{\alpha}^{\ell},-1\right)$. Ou seja, haverá arbitragem vendendo ou comprando o contrato. Suponha que tenhamos a estratégia da primeira forma $\Gamma^{\prime}=$ $\left(\bar{\beta}, \bar{\alpha}^{1}, \ldots, \bar{\alpha}^{\ell}, 1\right)$. Lembramos que $\Gamma$ é a cobertura do contrato $F$. Considere o portfólio

$$
\tilde{\Gamma}:=\left(\bar{\beta}, \bar{\alpha}^{1}, \ldots, \bar{\alpha}^{\ell}\right)+\Gamma
$$

no mercado $M_{n}$. Daí, $V_{0}^{\Gamma^{\prime}}=V_{0}^{\tilde{\Gamma}}$ e $V_{1}^{\Gamma^{\prime}}=V_{1}^{\tilde{\Gamma}}$, pois $\Gamma$ é cobertura de $F$. Daí se $\Gamma^{\prime}$ é oportunidade de arbitragem em $M_{n}^{\prime}$ teremos que $\tilde{\Gamma}$ será oportunidade de arbitragem em $M_{n}$, contradizendo a hipótese de não arbitragem. Agora se considerarmos uma estratégia da segunda forma, tomaríamos

$$
\tilde{\Gamma}:=\left(\bar{\beta}, \bar{\alpha}^{1}, \ldots, \bar{\alpha}^{\ell}\right)-\Gamma
$$

repetiríamos o argumento acima e obteríamos o mesmo resultado chegando a uma contradição.

Isso mostra que não pode haver arbitragem em $M_{n}^{\prime}$ e o único preço do contrato é igual a $P$.

A Proposição 2.10 mostrou que o preço de um contrato que possui cobertura deve ser igual ao preço do portfólio de cobertura em $n=0$. Isto vale para o caso de mercados completos, ou seja, aqueles em que os contratos possuem sempre um portfólio de cobertura. Se existem contratos que não possuem cobertura o processo de definição do preço correto parece inviável, mas na verdade é possível, porém o preço de tais contratos poderá ser qualquer valor dentro de um intervalo definido na reta real.

Definição 2.11 Em um mercado incompleto para um contrato $F$, podemos descrever os possíveis conjuntos de portfólios

$$
\begin{aligned}
& E_{-}(F):=\left\{\Gamma \in \mathbb{R}^{\ell+1} ; V_{1}^{\Gamma}(\omega) \leqslant F(\omega), \forall \omega \in \Omega\right\} \\
& E_{+}(F):=\left\{\Gamma \in \mathbb{R}^{\ell+1} ; V_{1}^{\Gamma}(\omega) \geqslant F(\omega), \forall \omega \in \Omega\right\}
\end{aligned}
$$

Ou seja, $E_{-}(F)$ é o conjunto dos portfólios que em $n=1$ possuem valor menor ou igual ao valor do contrato e $E_{+}(F)$ são os que possuem valor maior ou igual. 
Definição 2.12 Em um mercado incompleto definimos para cada contrato $F$ os seguintes valores:

$$
\begin{aligned}
& p_{-}(F):=\sup \left\{V_{0}^{\Gamma} ; \Gamma \in E_{-}(F)\right\}, \\
& p_{+}(F):=\inf \left\{V_{0}^{\Gamma} ; \Gamma \in E_{+}(F)\right\} .
\end{aligned}
$$

Definindo os valores $p_{-}(F)$ e $p_{+}(F)$, temos como objetivo encontrar uma faixa de preços, ou seja, um intervalo, em que seja possível precificar o contrato $F$.

Proposição 2.13 Se $\Omega$ é finito e nosso mercado é livre de arbitragem e incompleto então existem portfólios $\Gamma_{+} \in E_{+}(F)$ e $\Gamma_{-} \in E_{-}(F)$ tais que $p_{-}(F)=V_{0}^{\Gamma_{-}}$e $p_{+}(F)=V_{0}^{\Gamma_{+}}$.

Para provar esta proposição usaremos alguns fatos de Otimização Linear e o conceito de Probabilidade de Risco Neutro, que será apresentado e detalhado no Capítulo 3.

Como veremos à frente no texto, o fato do mercado ser livre de arbitragem implica a existência de alguma probabilidade neutra ao risco. O fato do mercado ser incompleto e livre de arbitragem implica que existem diversas probabilidades neutras ao risco. Um contrato possui um preço correto se para qualquer probabilidade neutra ao risco obtemos o mesmo preço para ele em $n=0$.

Prova. Seja $F$ o contrato que queremos precificar. Supomos que está em um mercado incompleto e que não exista somente um preço correto em $n=0$ para um contrato. Lembramos que $B_{1}$ é o preço do ativo livre de risco em $n=1$, ou seja, $B_{1}=(1+r)$. Tomemos $\Gamma \in E_{+}(F)$. Para qualquer probabilidade neutra ao risco $\mathbb{Q}$ teremos

$$
\mathbb{E}_{\mathbb{Q}}\left(\frac{V_{1}^{\Gamma}}{B_{1}}\right)=V_{0}^{\Gamma} \text { e } \quad \mathbb{E}_{\mathbb{Q}}\left(\frac{V_{1}^{\Gamma}}{B_{1}}\right) \geqslant p_{+}(F)
$$

Além disso, haverá um limite inferior para o conjunto $p_{+}(F)$ definido por $\sup \left\{\mathbb{E}_{\mathbb{Q}}\left(\frac{F}{B_{1}}\right) ; \mathbb{Q}\right.$ é Probabilidade Neutra ao Risco $\}$. Agora sabendo que $\Omega$ é finito, notemos que $p_{+}(F)$ pode ser interpretado como a solução de um problema de otimização linear

$$
\begin{aligned}
& \text { minimizar } V_{0}^{\Gamma} \\
& \text { sujeito a } V_{1}^{\Gamma} \geqslant F \\
& \Gamma \in \mathbb{R}^{\ell+1} .
\end{aligned}
$$


Como estamos minimizando nosso problema em uma região factível não vazia e como a função objetivo é limitada por baixo, como foi mostrado, então o valor ótimo é atingido, isso pode ser visto em maiores detalhes em [1]. Concluímos que $p_{+}(F)=V_{0}^{\Gamma_{+}}$para um $\Gamma_{+} \in E_{+}$.

O mesmo procedimento realizado a $p_{-}(F)$, porém maximizando, nos levará à existência de $p_{-}(F)=V_{0}^{\Gamma_{-}}$para um $\Gamma_{-} \in E_{-}$, e concluímos a demonstração.

As próximas proposições nos dirão que um contrato que não possua cobertura num mercado incompleto terá um intervalo de preços corretos.

Proposição 2.14 Seja F um contrato em um mercado livre de arbitragem $M_{n}$. Para qualquer par de portfolios $\Gamma^{(1)} \in E_{-}(F)$ e $\Gamma^{(2)} \in E_{+}(F)$ temos $V_{0}^{\Gamma^{(1)}} \leqslant V_{0}^{\Gamma^{(2)}}$ e portanto, $p_{-}(F) \leqslant p_{+}(F)$.

Prova. Demonstraremos usando basicamente o conceito de mercado livre de arbitragem. Tome o portfólio $\Gamma^{\prime}=\Gamma^{(2)}-\Gamma^{(1)}$. Sabemos das definições que $V_{1}^{\Gamma^{(1)}} \leqslant F \leqslant V_{1}^{\Gamma^{(2)}}$, então $V_{1}^{\Gamma^{2}}-V_{1}^{\Gamma^{1}} \geqslant 0$, e por isso $V_{1}^{\Gamma^{\prime}} \geqslant 0$. Usando o fato de $M_{n}$ ser um mercado livre de arbitragem, devemos ter $V_{0}^{\Gamma^{\prime}} \geqslant 0$ e temos nosso resultado. Se fosse $V_{0}^{\Gamma^{\prime}}<0$ nosso mercado ofereceria uma oportunidade de arbitragem. Se fizermos $\Gamma^{2}=\Gamma^{+}$e $\Gamma^{1}=\Gamma_{-}$teremos que $V_{0}^{\Gamma_{(-)}} \leqslant V_{0}^{\Gamma_{(+)}}$e como $p_{-}(F)=V_{0}^{\Gamma_{-}}$e $p_{+}(F)=V_{0}^{\Gamma_{+}}$concluímos que $p_{-}(F) \leqslant p_{+}(F)$.

Proposição 2.15 Um contrato $F$ possui cobertura se e somente se $p_{-}(F)=$ $p_{+}(F)$. Se $\Gamma$ é uma cobertura de $F$ então $p_{-}(F)=V_{0}^{\Gamma}=p_{+}(F)$.

Prova. Primeiro vamos supor que $F$ possui cobertura. Então sabemos que $\exists \Gamma ; V_{1}^{\Gamma}(\omega)=F(\omega), \forall \omega \in \Omega$. Logo $\Gamma \in E_{-}(F) \cap E_{+}(F)$. Pela definição de $p_{-}(F)$ e $p_{+}(F)$ temos $p_{+}(F) \leqslant V_{0}^{\Gamma} \leqslant p_{-}(F)$. Como na Proposição 2.14 temos $p_{-}(F) \leqslant p_{+}(F)$ concluímos que $p_{-}(F)=p_{+}(F)$.

Agora vamos supor que $p_{-}(F)=p_{+}(F)$. Consideremos o portfólio $\Gamma^{\prime}=$ $\Gamma_{+}-\Gamma_{-}$, com $\Gamma_{+} \in E_{+}(F)$ e $\Gamma_{-} \in E_{-}(F)$ que sabemos que existem, onde $p_{-}(F)=V_{0}^{\Gamma_{-}}$e $p_{+}(F)=V_{0}^{\Gamma_{+}}$. Agora temos que $V_{0}^{\Gamma^{\prime}}=V_{0}^{\Gamma_{+}}-V_{0}^{\Gamma_{-}}$e pela hipótese que assumimos temos $V_{0}^{\Gamma^{\prime}}=0$. Como o mercado é livre de arbitragem, devemos ter $V_{1}^{\Gamma^{\prime}}=0$ o que implica em $V_{1}^{\Gamma_{+}}=V_{1}^{\Gamma_{-}}=F$. Concluímos que $V_{0}^{\Gamma_{+}}=V_{0}^{\Gamma_{-}}$e também que se chamarmos a cobertura de $\Gamma$ então $p_{-}(F)=V_{0}^{\Gamma}=p_{+}(F)$.

Depois de apresentarmos alguns resultados sobre desigualdades de preços devido a portfólios que não são coberturas exatas de um contrato vamos 
mostrar que o preço de um contrato sem cobertura deve estar contido em um intervalo específico para que não haja oportunidade de arbitragem no mercado.

Proposição 2.16 Seja $M_{n}$ um mercado sem arbitragem e $F$ um contrato que não possui cobertura. Então o conjunto de preços corretos para $F$ é o intervalo aberto $\left(p_{-}(F), p_{+}(F)\right)$.

Prova. Consideremos o mercado $M_{n}^{\prime}$ onde o contrato é um ativo de risco. Suponhamos que o preço $P$ do contrato fosse $P \leqslant p_{-}(F)$. Faz sentido vender o contrato no mercado pelo preço $p_{-}(F)=V_{0}^{\Gamma_{-}}$e adquirirmos o mesmo contrato ao preço $P$. Por isso, tomamos o portfólio $\Gamma^{\prime}=\left(-\Gamma_{-}, 1\right)$ em $M_{n}^{\prime}$, onde $\Gamma_{-}$é o portfólio que tem como valor $V_{0}^{\Gamma_{-}}$segundo a Proposição 2.13. Então

$$
\begin{aligned}
V_{0}^{\Gamma^{\prime}} & =\Gamma^{\prime} \cdot M_{0}^{\prime}=-V_{0}^{\Gamma_{-}}+P \\
& =-p_{-}(F)+P \leqslant 0
\end{aligned}
$$

logo, o agente embolsa uma quantia positiva em $n=0$. Quando $n=1$, temos

$$
V_{1}^{\Gamma^{\prime}}=-V_{1}^{\Gamma_{-}}+F \geqslant 0
$$

pois $\Gamma_{-} \in E_{-}(F)$. Já que $F$ não possui uma cobertura, $\Gamma^{\prime}$ é uma oportunidade de arbitragem em $M_{n}^{\prime}$ e consequentemente $P$ não é o preço correto.

Agora seja $P \geqslant p_{+}(F)$ e tomemos o portfólio $\Gamma^{\prime \prime}=\left(\Gamma_{+},-1\right)$, onde $\Gamma_{+}$é o portfólio que tem como valor $V_{0}^{\Gamma_{+}}$segundo a Proposição 2.13. Vendemos o contrato ao preço $P$ e compramos a $p_{+}(F)$. Temos

$$
\begin{aligned}
V_{0}^{\Gamma^{\prime \prime}} & =\Gamma^{\prime \prime} \cdot M_{0}^{\prime}=V_{0}^{\Gamma_{+}}-P \\
& =p_{+}(F)-P \leqslant 0
\end{aligned}
$$

Quando $n=1$, temos

$$
V_{1}^{\Gamma^{\prime \prime}}=V_{1}^{\Gamma_{+}}-F \geqslant 0
$$

pois $\Gamma_{+} \in E_{+}(F)$. Concluímos que também há arbitragem.

Agora, devemos mostrar que $P \in\left(p_{-}(F), p_{+}(F)\right)$ não acarreta arbitragem em $M_{n}^{\prime}$.

Se $p_{-}(F)<P<p_{+}(F)$ pode-se tentar arbitrar de duas formas em $M_{n}^{\prime}$ :

1. Tomando $\Gamma^{\prime}=\left(\Gamma_{-},-1\right)$, ou seja, vendendo o contrato ao preço $P$ e adquirindo o portfólio $\Gamma_{-}$;

$\mathrm{Ou}$ 
2. Tomando $\Gamma^{\prime \prime}=\left(-\Gamma_{+}, 1\right)$, vendendo o portfólio $\Gamma_{+}$e adquirindo o contrato a $P$.

Realizando o mesmo procedimento acima, quando verificamos que haveria arbitragem, é rápido ver que não há arbitragem em $\left(p_{-}(F), p_{+}(F)\right)$, pois sempre teremos $V_{1}^{\Gamma_{-}}(\omega) \leqslant F(\omega) \leqslant V_{1}^{\Gamma_{+}}(\omega) \forall \omega \in \Omega$, ou seja, não há como obter um ganho certo, pois o agente tem a possibilidade de não conseguir fechar suas posições sem que haja perda financeira. Desta forma, este é o intervalo de preços correto.

\section{7}

\section{Um mercado com vários períodos de tempo.}

O modelo considerado até aqui era dotado de $\ell+1$ ativos, sendo um ativo livre de risco e $\ell$ ativos com risco. Havia somente os períodos de tempo $n=0$ e $n=1$, enquanto que agora iremos considerar o tempo variando de 0 a $N$, com $N$ finito. O intervalo temporal será finito, pois estamos interessados na dinâmica de contratos com uma data de liquidação pré estabelecida.

Definição 2.17 Chamamos de mercado financeiro um processo estocástico (a definição de processo estocástico pode ser consultada na Definição A.11) de $\ell+1$ variáveis $M_{n}=\left(B_{n}, S_{n}^{1}, \ldots, S_{n}^{\ell}\right) ; n \in\{0,1, \ldots N\}$ definido sobre o espaço filtrado $\left(\Omega, \mathcal{F}_{n}\right)$, onde $\left\{\mathcal{F}_{n}\right\}, n \in\{1,2, \ldots N\}$, de maneira que:

- $\Omega$ é finito e $\mathcal{F}_{N}=\mathcal{F}$, onde $\mathcal{F}$ é a $\sigma$-álgebra da definição do nosso espaço de probabilidade.

- $B_{n}$ é determinístico, com $B_{0}=1, B_{n}=\left(1+r_{n}\right) B_{n-1}$, onde $\left\{r_{n} ; n \in \mathbb{N}\right\}$ é uma sequência de números reais.

- Em $n=0$ o mercado é determinístico: $M_{0}=\left(B_{0}, S_{0}^{1}, \ldots S_{0}^{\ell}\right)$ é constante.

- A filtração $\left\{\mathcal{F}_{n}\right\}, n \in\{0,1, \ldots N\}$ é gerada pela sequência dos processos $M_{n}$

$$
\mathcal{F}_{n}:=\sigma\left(M_{0}, M_{1}, \ldots M_{n}\right)
$$

ou seja, é a $\sigma$-álgebra gerada pelas variáveis aleatórias $M_{0}, M_{1}, \ldots M_{n}$. A definição de $\sigma$-álgebra gerada por variáveis aleatórias pode ser encontrada na Definição A.5.

$B_{n}$ é nosso ativo livre de risco que tem seu valor variando de $n$ a $n+1$ de acordo com a taxa de juros $r_{n}$. Nossos ativos com risco, representados por $S_{n}^{i}$ com $i$ estando entre 1 e $\ell$, são variáveis aleatórias definidas por $S^{i}: \Omega \longrightarrow \mathbb{R}$. 
A dinâmica da informação é dada pela filtração originada da sequência de préimagens de $M_{n}$, pois $\mathcal{F}_{n}$ é formado pelas pré-imagens $M_{j}^{-1}(B), j \in\{0,1, \ldots, n\}$ e $B \in \mathcal{B}$, onde $\mathcal{B}$ é a $\sigma$-álgebra de Borel em $\mathbb{R}$. A cada nova observação do estado do mercado obtemos mais informação que é agregada à $\sigma$-álgebra $\mathcal{F}_{n}$ respectiva aquele período $n$ de tempo.

Em um mercado a vários períodos de tempo a definição de estratégia é a mesma para o mercado a dois períodos de tempo, com a diferença de que o agente pode realocar as proporções dos ativos entre um período de tempo e outro, segundo informações disponíveis e segundo sua restrição de recursos.

Definição 2.18 Uma estratégia $\Gamma_{n}=\left(\beta_{n}, \alpha_{n}^{1}, \alpha_{n}^{2}, \ldots, \alpha_{n}^{\ell}\right), n \in\{1,2, \ldots, N\}$ é um processo estocástico previsível (a definição de processo previsível pode ser acessada na Definição A.12) de $\ell+1$ variáveis todas definidas sobre o mesmo espaço filtrado do mercado.

No período de tempo $n$ o agente tem suas posições no mercado identificadas pelo vetor $\Gamma_{n}$. Segundo as informações disponíveis até o instante de tempo atual, representadas por $\mathcal{F}_{n}$, o agente pode tomar decisões de alterar sua estratégia para $\Gamma_{n+1}$ reorganizando as quantidades dos ativos que constituem seu portfólio. O agente toma essa decisão baseado na informação em $n$, por isso fazemos a estratégia $\Gamma_{n+1}$ ser um processo previsível (ver Definição A.12). Além disso, desejamos que durante a alteração da estratégia entre o tempo $n$ e $n+1$ o agente não realize nenhuma aplicação de capital, ou seja, que o valor das estratégias seja o mesmo quando o agente trocar de uma para outra. Daí que introduzimos a seguinte definição.

Definição 2.19 Uma estratégia $\Gamma_{n}$ é auto-financiada quando

$$
\Gamma_{n} \cdot M_{n}=\Gamma_{n+1} \cdot M_{n}
$$

para $n \in\{1,2, \ldots, N-1\}$.

Definição 2.20 A cada estratégia $\Gamma_{n}$ associaremos o processo $V_{n}^{\Gamma}$. Dessa maneira, $V_{0}^{\Gamma}=\Gamma_{0} \cdot M_{1}$ e $V_{n}^{\Gamma}=\Gamma_{n} \cdot M_{n}, \forall n \in\{1,2, \ldots, N\}$.

Vemos que $V_{0}^{\Gamma}$ é determinístico e é a quantia necessária para iniciar a estratégia $\Gamma_{0}$. Enquanto que $V_{n}^{\Gamma}$ é a quantia obtida quando há a liquidação da estratégia $\Gamma_{n}$.

Proposição 2.21 Uma estratégia é auto-financiada se e somente se

$$
V_{m}^{\Gamma}-V_{n}^{\Gamma}=\int_{n}^{m} \Gamma d M
$$

para todo $0 \leqslant n \leqslant m \leqslant N$. 
A integral usada acima é definida na Definição A.13, onde apresentamos a transformada martingal. A integral é uma notação para a transformada martingal.

Prova. Primeiramente, vamos supor que $\Gamma_{n}$ é auto-financiada. Podemos escrever $V_{n}^{\Gamma}-V_{0}^{\Gamma}$ como a soma telescópica $\sum_{i=1}^{n}\left(V_{i}^{\Gamma}-V_{i-1}^{\Gamma}\right)$. Usando o fato da estratégia $\Gamma_{n}$ ser auto-financiada (Definição 2.19) desenvolvemos a soma como

$$
\begin{aligned}
\sum_{i=1}^{n}\left(V_{i}^{\Gamma}-V_{i-1}^{\Gamma}\right) & =\sum_{i=1}^{n}\left(\Gamma_{i} \cdot M_{i}-\Gamma_{i} \cdot M_{i-1}\right) \\
& =\sum_{i=1}^{n} \Gamma_{i} \cdot\left(M_{i}-M_{i-1}\right)
\end{aligned}
$$

que é exatamente a integral definida na Definição A.13. Assim mostramos a implicação. Para mostrar a equivalência vejamos que

$$
V_{n+1}^{\Gamma}-V_{n}^{\Gamma}=\Gamma_{n+1} \cdot\left(M_{n+1}-M_{n}\right)
$$

que existe diretamente da definição da integral. Logo

$$
\Gamma_{n+1} \cdot M_{n+1}-\Gamma_{n} \cdot M_{n}=\Gamma_{n+1} \cdot M_{n+1}-\Gamma_{n+1} \cdot M_{n}
$$

onde escrevemos todos os termos como produtos escalares. Daqui resulta que

$$
\Gamma_{n} \cdot M_{n}=\Gamma_{n+1} \cdot M_{n}
$$

Assim, concluímos a prova e temos a equivalência.

Neste contexto, a definição de arbitragem é similar à definição no caso em que o modelo tem dois períodos de tempo, com a óbvia mudança de que a data final passa a ser $N$ ao invés de 1 e a estratégia que pode gerar arbitragem é auto-financiada, já que agora iremos nos restringir somente a este tipo de estratégia. Relembrando o que já vimos neste contexto, uma oportunidade de arbitragem é a existência de uma sequência de estratégias auto-financiadas que resultem em uma probabilidade positiva de ganhar em $N$ e extinguam as chances de haver alguma perda financeira.

A mudança na data final do modelo se aplica aos contratos, pois agora a data de vencimento deles é a última data do modelo.

Definição 2.22 Seja $M_{n}$ um mercado sem arbitragem e $F: \Omega \longrightarrow \mathbb{R}$ um contrato. Considere o espaço mensurável $(\Omega, \mathcal{F})$, a filtração $\left\{\mathcal{F}_{n}\right\}, n \in$ $\{1,2, \ldots N\}, P_{n}(F)$ definido sobre o espaço filtrado descrito é um processo de preços corretos para $F$ se 
1. $P_{N}(F)=F$,

2. O mercado $M_{n}^{\prime}:=\left(B_{n}, S_{n}, P_{n}(F)\right)$ é livre de arbitragem.

onde $B_{n}$ e $S_{n}$ são os preços do ativo livre de risco e do ativo com risco, respectivamente, no período $n \in\{1,2, \ldots N\}$,

Definir o processo dos preços do contrato como uma variável $\mathcal{F}_{n}$-mensurável faz sentido, pois em $n$ haverá a informação a respeito do valor de $P_{n}(F)$. Definir o novo mercado como sendo livre de arbitragem garante que não há a possibilidade de realizar lucros sem risco.

O seguinte lema esclarece outra forma de enxergarmos arbitragem realizada por uma estratégia no mercado. Note que as quantidades de cada ativo que compõe o portfólio são agora ajustáveis no tempo, ou seja, $\alpha_{n}$ e $\beta_{n}$ representam as quantidades de cada ativo no período $n \in\{1,2, \ldots, N\}$.

Lema 2.23 Se no mercado $M_{n}$ existe uma estratégia $\Gamma_{n}=\left(\alpha_{n}, \beta_{n}\right)$ tal que $V_{0}^{\Gamma}<0$ e $V_{N}^{\Gamma}=0$ então $M_{n}$ não é livre de arbitragem.

Prova. Escrevemos $V_{0}^{\Gamma}=\left(\beta_{1}, \alpha_{1}\right) \cdot M_{0}<0$. Consideremos a estratégia $\tilde{\Gamma}_{n}=\left(\beta_{n}-V_{0}^{\Gamma}, \alpha_{n}\right)$ de forma que $V_{0}^{\tilde{\Gamma}}=0 . \operatorname{Em} n=N$ teremos $V_{N}^{\tilde{\Gamma}}=\beta_{N} B_{N}+$ $\alpha_{N} S_{N}-V_{0}^{\Gamma} B_{N}$. Como $V_{N}^{\Gamma}=0$ por hipótese, ficamos com $V_{N}^{\tilde{\Gamma}}=-V_{0}^{\Gamma} B_{N}>0$. Portanto esta é uma estratégia usada para arbitrar em $M_{n}$.

Proposição 2.24 Seja o mercado $M_{n}$ livre de arbitragem e um contrato $F: \Omega \longrightarrow \mathbb{R}$. Se F possui um portfólio auto-financiado de cobertura, ou seja, $V_{N}(\omega)=F(\omega) \forall \omega \in \Omega$, então $F$ possui um único processo de preços corretos $P_{n}(F)$. Além disso,

$$
P_{n}(F)=V_{n}^{\Gamma}, \forall n \in\{1,2, \ldots N\}
$$

Prova. Primeiro vamos mostrar que $V_{n}^{\Gamma}$ é um processo de preços corretos para $F$. Para isso vamos começar mostrando que o mercado $M_{n}^{\prime}=\left(B_{n}, S_{n}, V_{n}^{\Gamma}\right)$ é livre de arbitragem. Suponha que exista uma estratégia $\Gamma^{\prime}=\left(\beta_{n}^{\prime}, \alpha_{n}^{\prime}, \theta_{n}^{\prime}\right), \theta_{n}^{\prime}$ sendo a quantidade do ativo contrato presente no portfólio, gerando arbitragem em $M_{n}^{\prime}$. Logo

$$
\begin{aligned}
V_{n}^{\Gamma^{\prime}} & =\Gamma_{n}^{\prime} \cdot M_{n}^{\prime} \\
& =\left(\beta_{n}^{\prime}, \alpha_{n}^{\prime}, \theta_{n}^{\prime}\right) \cdot\left(B_{n}, S_{n}, V_{n}^{\Gamma}\right) \\
& =\left(\beta_{n}^{\prime}, \alpha_{n}^{\prime}\right) \cdot\left(B_{n}, S_{n}\right)+\theta_{n}^{\prime} \Gamma_{n} \cdot M_{n}
\end{aligned}
$$


o termo $\theta^{\prime} \Gamma_{n} \cdot M_{n}$ pode ser escrito como o valor de um portfólio $\theta^{\prime} \Gamma_{n}$, ou seja, a quantia de $V_{n}^{\theta^{\prime} \Gamma}$ unidades de ativo livre de risco no período $n$. Desta maneira, com $M_{n}=\left(B_{n}, S_{n}\right)$ temos que

$$
\begin{aligned}
V_{n}^{\Gamma^{\prime}} & =\left(\beta_{n}^{\prime}, \alpha_{n}^{\prime}\right) \cdot M_{n}+V_{n}^{\theta^{\prime} \Gamma} \\
& =V_{n}^{\theta^{\prime} \Gamma+\left(\beta_{n}^{\prime}, \alpha_{n}^{\prime}\right)}
\end{aligned}
$$

chegamos a uma estratégia que é arbitragem em $M_{n}$, uma contradição com a hipótese de que $M_{n}$ é livre de arbitragem.

Agora devemos mostrar que se $P_{0}(F)>V_{0}^{\Gamma}$ ou $P_{0}(F)<V_{0}^{\Gamma}$ teremos que $P_{n}(F)$ não pode ser um processo de preços correto. Em outras palavras, $V_{n}^{\Gamma}$ tem que ser o único processo de preços correto. Se $P_{0}(F)>V_{0}^{\Gamma}$ então considere a estratégia $\Gamma_{n}^{\prime}=\left(\Gamma_{n},-1\right)$ em $M_{n}^{\prime}$, em que um agente vende um contrato ao preço $P_{0}(F)$, que está caro e adquire o portfólio de cobertura $\Gamma$, que está barato. Assim, $V_{0}^{\Gamma^{\prime}}=\left(\alpha_{1}, \beta_{1}\right) \cdot\left(B_{0}, S_{0}\right)-P_{0}(F)=\Gamma_{1} \cdot M_{0}-P_{0}(F)<0$ e como é um portfólio de cobertura, teremos em $N$ que $V_{N}^{\Gamma^{\prime}}=\Gamma_{N} \cdot M_{N}-P_{N}(F)=$ 0 , mostrando que $M_{N}^{\prime}$ não é um mercado livre de arbitragem (arbitragem realizada segundo o Lema 2.23). Se $P_{0}(F)<V_{0}^{\Gamma}$ o agente pode tomar a estratégia $\Gamma_{n}^{\prime}=\left(-\Gamma_{n}, 1\right)$, em que vende o portfólio de cobertura $\Gamma$ (o agente toma o portfólio emprestado e vende, mas no futuro terá obrigação que retornar o que tomou emprestado) e adquire o contrato ao preço $P_{0}(F)$ o que mostrará que $V_{0}^{\Gamma^{\prime}}=\Gamma_{1} \cdot M_{0}-P_{0}(F)>0$ e $V_{N}^{\Gamma^{\prime}}=-\Gamma_{N} \cdot M_{N}+P_{N}(F)=0$, ou seja, também há arbitragem. Provando estes pontos concluímos que $P_{n}(F)$ deve ser um processo de preços correto.

Neste capítulo, buscamos introduzir os conceitos usados na construção de um modelo de mercado, inicialmente simples e gradativamente incrementado. A noção de arbitragem e sua vital importância na precificação de contratos deve ser ressaltada. A definição de cobertura e a relação de completude em um mercado é outro ponto importante e leva a um tratamento mais delicado no caso de uma precificação em mercados incompletos. Por fim, a extensão a vários períodos de tempo exige um instrumental de processos estocásticos que permitem um tratamento do fluxo de informações. As definições básicas se mantêm quase as mesmas do modelo mais simples ao último apresentado e são ideias fundamentais na modelagem financeira. 


\section{Resultados Fundamentais}

\section{1}

\section{Probabilidade Neutra ao Risco}

Nesta seção, introduziremos o conceito de Probabilidade Neutra ao Risco. Com a probabilidade neutra ao risco será possível obter informações mais precisas a respeito do mercado, sua completude e, principalmente, sob que condições haverá a correta precificação de um contrato.

Consideremos o mercado formado por $\ell+1$ ativos e dois períodos de tempo

$$
M_{n}=\left(B_{n}, S_{n}^{1}, \ldots S_{n}^{\ell}\right) \text { com n } \in\{0,1\}
$$

definido sobre o conjunto $\Omega=\left\{\omega_{1}, \omega_{2}, \ldots \omega_{k}\right\}$ finito. Consideremos agora para cada ativo do mercado o seu preço descontado, ou seja, a razão entre o preço do ativo no período $n$ sobre o preço de outro ativo no mesmo período. Em geral tomamos o preço $B_{n}$ do ativo livre de risco como fator de desconto e temos a seguinte definição

$$
\overline{S_{n}^{i}}=\frac{S_{n}^{i}}{B_{n}}
$$

para $i \in\{1,2, \ldots \ell\}$.

Descontando o preço de cada ativo no mercado $M_{n}$, temos o mercado $\overline{M_{n}}=\left(1, \overline{S_{n}^{1}}, \overline{S_{n}}, \ldots \overline{S_{n}^{\ell}}\right)$, ou seja, o mercado $M_{n}$, porém normalizado pelo ativo $B_{n}$.

Definição 3.1 Seja $\mathbb{P}$ uma medida de probabilidade sobre o espaço $\Omega$ finito tal que $\mathbb{P}(\omega)>0 \forall \omega \in \Omega$. Então $\mathbb{P}$ é uma probabilidade neutra ao risco para o mercado $M_{n}$ se para cada ativo $i$ do mercado vale $\mathbb{E}_{\mathbb{P}}\left(\overline{S_{1}^{i}}\right)=\overline{S_{0}^{i}}$.

Em outras palavras, considerando o valor do ativo livre de risco em $n=0$ como sendo $B_{0}=1$, o que a definição acima diz é que sob a medida de probabilidade $\mathbb{P}$, o retorno esperado do ativo com risco deve ser igual ao retorno do ativo livre de risco: $\mathbb{E}_{\mathbb{P}}\left(S_{1}^{i}\right)=(1+r) S_{0}^{i}$.

Convém notar que a probabilidade neutra ao risco é uma medida artificial de probabilidade, ou seja, em geral pode diferir da medida de probabilidade que os agentes atribuem, via inferência, a cada estado da natureza $\omega$. 
Em atuária existe um conceito conhecido como Princípio da Equivalência afirmando que para um ativo que tenha um valor futuro incerto ser precificado hoje, basta calcularmos o valor esperado do ativo e multiplicarmos por um fator de desconto para trazermos a valor presente. No caso de mercados financeiros isso pode acarretar problemas, pois agentes tem preferências individuais diferentes e tendem a exigir um retorno maior para um risco maior que assumem. Assim, quando os agentes calcularem o valor esperado do ativo com uma probabilidade obtida via inferência, estarão adicionando a aversão ao risco no cálculo do valor esperado e consequentemente o valor presente de determinado ativo será distinto no mercado. A solução para este problema surgiu com o conceito de probabilidade neutra ao risco. A probabilidade neutra ao risco é uma medida artificial criada para realizar cálculos como o dito acima de forma a desconsiderar as preferências de cada agente e, assim, obter o preço justo ou livre de arbitragem. A importância desta medida será apresentada nos enunciados dos teoremas abaixo e no restante do trabalho.

Os seguintes teoremas serão enunciados agora, mas suas demonstrações serão corolários de teoremas apresentados para mercados de $N$ períodos. Estes teoremas são vitais para o trabalho, por isso na sequência deste capítulo serão demonstrados e explicados em detalhes.

Teorema 3.2 Existe uma probabilidade neutra ao risco em $M_{n}$ se e somente se $M_{n}$ é um mercado livre de arbitragem.

Teorema 3.3 Suponha $M_{n}$ livre de arbitragem. $M_{n}$ é completo se e somente se existe uma única probabilidade neutra ao risco.

Para exemplificar o conceito de probabilidade neutra ao risco usaremos o primeiro modelo de mercado que analisamos, i.e, o mercado com dois ativos e dois períodos de tempo representado por $M_{n}=\left(B_{n}, S_{n}\right), n \in\{0,1\}$. Consideremos $\Omega=\left\{\omega_{1}, \omega_{2}\right\}$ e uma medida de probabilidade $\mathbb{P}$ estritamente positiva para os dois estados da natureza, i.e., $\mathbb{P}\left(\omega_{1}\right)>0$ e $\mathbb{P}\left(\omega_{2}\right)>0$. Sejam $(1+a)$ e $(1+b)$ os respectivos retornos do ativo de risco para $\omega_{1}$ e $\omega_{2}$, com $a<b$, e o retorno do ativo livre de risco como $(1+r)$.

Pela definição de probabilidade neutra ao risco apresentada anteriormente, notamos que $\mathbb{P}$ é uma probabilidade neutra ao risco se ao tomarmos o valor esperado pela medida $\mathbb{P}$

$$
\begin{aligned}
\mathbb{E}\left(\frac{S_{1}}{B_{1}}\right) & =\overline{S_{0}} \\
\mathbb{P}\left(\omega_{1}\right) \cdot \frac{S_{1}\left(\omega_{1}\right)}{B_{1}}+\mathbb{P}\left(\omega_{2}\right) \cdot \frac{S_{1}\left(\omega_{2}\right)}{B_{1}} & =\overline{S_{0}} \\
\frac{S_{0}(1+a)}{1+r} \mathbb{P}\left(\omega_{1}\right)+\frac{S_{0}(1+b)}{1+r} \mathbb{P}\left(\omega_{2}\right) & =\overline{S_{0}} .
\end{aligned}
$$


Na equação acima, faça $\mathbb{P}\left(\omega_{1}\right)=q$ e $\mathbb{P}\left(\omega_{2}\right)=1-q \operatorname{com} q \in(0,1)$. Agora, isolando $q$ na igualdade acima obteremos as probabilidades

$$
q=\frac{b-r}{b-a} ; 1-q=\frac{r-a}{b-a} .
$$

Notemos que $\mathbb{P}\left(\omega_{1}\right)>0$ e $\mathbb{P}\left(\omega_{2}\right)>0$, ou seja, $\mathbb{P}$ é probabilidade neutra ao risco, se e somente se $r \in(a, b)$ e a probabilidade neutra ao risco é única neste caso.

Então pelo Teorema 3.2 podemos afirmar que o mercado $M_{n}$ é livre de arbitragem se e somente se $r \in(a, b)$. Como obtemos $q$ acima de forma única, temos pelo Teorema 3.3 que o mercado $M_{n}$ é completo. Com este exemplo é possível entender a rápida aplicação e importância do conceito de probabilidade neutra ao risco aos mercados.

A relação desta medida com a precificação de contratos é direta e será apresentada em seguida.

No mercado $\overline{M_{n}}$ cada ativo tem seu preço descontado, então uma estratégia $\Gamma$ precificada neste mercado tem seu valor dado por

$$
\overline{V_{n}^{\Gamma}}=\Gamma \cdot \overline{M_{n}} .
$$

Como a probabilidade neutra ao risco vale para todos os ativos de risco do mercado, é natural desejar estender o conceito ao valor de um portfólio $\Gamma$. Assim, enunciamos a seguinte proposição:

Proposição 3.4 Seja $M_{n}$ um mercado livre de arbitragem e $\mathbb{P}$ uma probabilidade neutra ao risco então para qualquer portfólio $\Gamma$ vale $\mathbb{E}_{\mathbb{P}}\left(\overline{V_{1}^{\Gamma}}\right)=\overline{V_{0}^{\Gamma}}$.

Prova. Pela linearidade da esperança podemos escrever

$$
\begin{aligned}
\mathbb{E}_{\mathbb{P}}\left(\overline{V_{1}^{\Gamma}}\right) & =\mathbb{E}_{\mathbb{P}}\left(\Gamma \cdot \overline{M_{1}}\right) \\
& =\Gamma \cdot \mathbb{E}_{\mathbb{P}}\left(\overline{M_{1}}\right)
\end{aligned}
$$

Como $\overline{M_{1}}$ é um vetor de $\ell+1$ coordenadas, podemos aplicar a esperança a cada uma destas coordenadas e pelo que sabemos sobre probabilidade neutra ao risco (definição 3.1 ) obteremos $\mathbb{E}_{\mathbb{P}}\left(\overline{M_{1}}\right)=\overline{M_{0}}$. Assim temos que $\Gamma \cdot \mathbb{E}_{\mathbb{P}}\left(\overline{M_{1}}\right)=\Gamma \cdot \overline{M_{0}}=\overline{V_{0}^{\Gamma}} \cdot$ Logo, $\mathbb{E}_{\mathbb{P}}\left(\overline{V_{1}^{\Gamma}}\right)=\overline{V_{0}^{\Gamma}}$

Lembramos aqui que $B_{1}$ é o preço do ativo livre de risco em $n=1 \mathrm{com}$ taxa de juros $r$, i.e., $B_{1}=B_{0}(1+r)$. Se $B_{0}=1$ então $B_{1}=(1+r)$. 
Proposição 3.5 Seja $M_{n}$ um mercado livre de arbitragem e F um contrato. Se $\mathbb{P}$ é uma probabilidade neutra ao risco então

$$
P_{0}(F)=\mathbb{E}_{\mathbb{P}}\left(\frac{F}{B_{1}}\right)
$$

é um preço correto para $F$.

Prova. Vamos provar a implicação. Seja $M_{n}$ um mercado livre de arbitragem e $\mathbb{P}$ uma probabilidade neutra ao risco. Se $\mathbb{P}$ é a única probabilidade neutra ao risco então pelo Teorema 3.3 sabemos que $M_{n}$ é completo. Pela definição de mercado completo, existe uma estratégia $\Gamma$ tal que $V_{1}^{\Gamma}(\omega)=F(\omega) \forall \omega \in \Omega$. Portanto pela Proposição 3.4 sabemos que $\mathbb{E}_{\mathbb{P}}\left(\overline{V_{1}^{\Gamma}}\right)=\overline{V_{0}^{\Gamma}}$, ou seja, $\mathbb{E}_{\mathbb{P}}\left(\frac{F}{B_{1}}\right)=\overline{V_{0}^{\Gamma}}$. Usando o fato do mercado ser livre de arbitragem, temos $V_{0}^{\Gamma}=P_{0}(F)$. Como em geral, $B_{0}=1$, pois é tido como período base, concluímos que $P_{0}(F)=\mathbb{E}_{\mathbb{P}}\left(\frac{F}{B_{1}}\right)$.

Agora tomemos o caso em que o conjunto das probabilidades neutras ao risco não seja único. Neste caso o mercado é incompleto (Teorema 3.3). Consideremos $F$ um contrato que não possua cobertura. Pela Proposição 2.13 existem portfólios $\Gamma_{-}$e $\Gamma_{+}$tais que $V_{1}^{\Gamma_{-}}(\omega) \leqslant F(\omega) \leqslant V_{1}^{\Gamma_{+}}(\omega) \forall \omega \in$ $\Omega$. Pela Definição 3.1 sabemos que quando calculamos o valor esperado usando qualquer medida de probabilidade neutra ao risco, neste caso uma probabilidade neutra ao risco $\mathbb{Q}$, obtemos $V_{0}^{\Gamma_{-}}=\mathbb{E}_{\mathbb{Q}}\left(\frac{V_{1}^{\Gamma_{-}}}{B_{1}}\right)$ e $V_{0}^{\Gamma_{+}}=\mathbb{E}_{\mathbb{Q}}\left(\frac{V_{1}^{\Gamma_{+}}}{B_{1}}\right)$ novamente considerando $B_{0}=1$. Pela Proposição 2.13 e pela Proposição 2.16,

$$
p_{-}(F)=V_{0}^{\Gamma_{-}}<p_{+}(F)=V_{0}^{\Gamma_{+}},
$$

pois $F$ não possui cobertura. Isto quer dizer que tomando o valor esperado sob uma probabilidade neutra ao risco obteremos um preço no intervalo de preços. $P_{0}(F)=\mathbb{E}_{\mathbb{Q}}\left(\frac{F}{B_{1}}\right) \in\left(p_{-}(F), p_{+}(F)\right)$ é um preço correto.

Usando o que já foi discutido a respeito de precificação de contratos, sabemos que se o contrato possui cobertura então o conjunto dos preços corretos deve ser unitário. Se o contrato $F$ não possui cobertura, então o conjunto dos preços corretos do contrato deve ser o intervalo de preços $\left(P_{-}(F), P_{+}(F)\right)$.

Como exemplo de precificação de contratos, podemos voltar ao que apresentamos no Capítulo 2, em que havia uma estratégia $\Gamma=(\beta, \alpha)$ que cobria um contrato $F$ no mercado $M_{n}=\left(B_{n}, S_{n}\right)$. Havia dois estados possíveis da natureza e os retornos do ativo com risco naquele cenário eram $(1+a)$ e $(1+b)$. Os preços do contrato em $t=1$ poderiam ser $F\left(\omega_{1}\right)=f_{1}$ e $F\left(\omega_{2}\right)=f_{2}$. 
O preço correto do contrato $F$ que calculamos foi

$$
P=\frac{f_{2}(r-a)+f_{1}(b-r)}{(1+r)(b-a)}
$$

Para este mercado já calculamos as probabilidades neutras ao risco nas equações $q=\frac{b-r}{b-a} ; 1-q=\frac{r-a}{b-a}$. Usando o Teorema anterior, obtemos

$$
\begin{aligned}
P_{0}(F) & =\mathbb{E}_{\mathbb{P}}\left(\frac{F}{B_{1}}\right) \\
& =\frac{\mathbb{P}\left(\omega_{1}\right) \cdot f_{1}}{(1+r)}+\frac{\mathbb{P}\left(\omega_{2}\right) \cdot f_{2}}{(1+r)} \\
& =\frac{(b-r) \cdot f_{1}}{(b-a)(1+r)}+\frac{(r-a) \cdot f_{2}}{(b-a)(1+r)} \\
& =\frac{f_{2}(r-a)+f_{1}(b-r)}{(1+r)(b-a)}=P
\end{aligned}
$$

que é exatamente o que havíamos mostrado sem esta ferramenta.

\section{2}

\section{Martingais}

Os martingais ocupam um papel central em nossa maneira de modelar a dinâmica dos ativos. Apresentaremos aqui as definições e proposições necessárias para a construção que se segue.

Definição 3.6 Seja $(\Omega, \mathcal{F}, \mathbb{P})\left\{\mathcal{F}_{n} ; n \in \mathbb{N}\right\}$ um espaço de probabilidade filtrado $e\left\{X_{n} ; n \in \mathbb{N}\right\}$ um processo estocástico assumindo valores em $\mathbb{R}$. A sequência $\left\{X_{n} ; n \in \mathbb{N}\right\}$ é dita martingal (relativa ao filtro $\left\{\mathcal{F}_{n} ; n \in \mathbb{N}\right\}$ ) quando

- $\mathbb{E}\left(\left|X_{n}\right|\right)<\infty \forall n \in \mathbb{N}$

- $\left\{X_{n} ; n \in \mathbb{N}\right\}$ é $\mathcal{F}_{n}$-mensurável $\forall n \in \mathbb{N}$;

$-\mathbb{E}\left(X_{n+1} \mid \mathcal{F}_{n}\right)=X_{n} \forall n \in \mathbb{N}$.

Proposição 3.7 Se $\left\{X_{n}, \mathcal{F}_{n} ; n \in \mathbb{N}\right\}$ é um martingal, então valem as seguintes afirmações para todo $n \in \mathbb{N}$ :

1. $\forall k \in \mathbb{N}, \mathbb{E}\left(X_{n+k} \mid \mathcal{F}_{n}\right)=X_{n}$;

2. $\mathbb{E}\left(X_{n}\right)=\mathbb{E}\left(X_{0}\right)$.

Prova. A primeira afirmação é demonstrada por indução em $k$. Por definição de martingal a afirmação, é válida para $k=1$. Suponha válida para $k_{0}$. Então, 
usando o ponto 7 da Proposição A.20

$$
\begin{aligned}
\mathbb{E}\left(X_{n+k_{0}+1} \mid \mathcal{F}_{n}\right) & =\mathbb{E}\left(\mathbb{E}\left(X_{n+k_{0}+1} \mid \mathcal{F}_{n+k_{0}}\right) \mid \mathcal{F}_{n}\right) \\
& =\mathbb{E}\left(X_{n+k_{0}} \mid \mathcal{F}_{n}\right)=X_{n}
\end{aligned}
$$

onde usamos a hipótese de que era válido para $k_{0}$ na segunda igualdade.

A segunda afirmação é demonstrada aplicando a esperança em ambos os lados da primeira igualdade e usando o ponto 9 da Proposição A.20 temos que

$$
\begin{aligned}
\mathbb{E}\left(\mathbb{E}\left(X_{n+k} \mid \mathcal{F}_{n}\right)\right) & =\mathbb{E}\left(X_{n}\right) \\
\mathbb{E}\left(X_{n+k}\right) & =\mathbb{E}\left(X_{n}\right)
\end{aligned}
$$

como vale para todo $n$ e $k$, temos nosso resultado.

Lema 3.8 Seja $\left\{X_{n}, \mathcal{F}_{n} ; n \in \mathbb{N}\right\}$ um martingal e $X$ uma variável aleatória integrável, ambos definidos no mesmo espaço de probabilidade. Afirmamos que

1. Se $X_{n}$ é um martingal não necessariamente de média zero então $M_{n}=$ $X_{n}-\mathbb{E}\left(X_{n}\right)$ é um martingal de média zero;

2. $\left\{Y_{n}, \mathcal{F}_{n} ; n \in \mathbb{N}\right\}$ é um martingal onde $Y_{n}=\mathbb{E}\left(X \mid \mathcal{F}_{n}\right)$.

Prova. Para a primeira afirmação devemos verificar se $M_{n}$ cumpre as condições para ser um martingal. Note que $M_{n}$ é $\mathcal{F}_{n}$-mensurável, pois $X_{n}$ e $\mathbb{E}\left(X_{n}\right)$ o são. Por outro lado vemos que $X_{n}$ é integrável por ser um martingal. Falta verificarmos uma propriedade, ou seja

$$
\begin{aligned}
\mathbb{E}\left(M_{n+1} \mid \mathcal{F}_{n}\right) & =\mathbb{E}\left(X_{n+1}-\mathbb{E}\left(X_{n+1}\right) \mid \mathcal{F}_{n}\right) \\
& =X_{n}-\mathbb{E}\left(\mathbb{E}\left(X_{n+1} \mid \mathcal{F}_{n}\right)\right) \\
& =X_{n}-\mathbb{E}\left(X_{n}\right)=M_{n}
\end{aligned}
$$

onde usamos a linearidade da esperança condicional. Para a prova da segunda afirmação vejamos que $Y_{n}$ é integrável e $\mathcal{F}_{n}$-mensurável. Note que $\mathbb{E}\left(Y_{n}\right)=$ $\mathbb{E}(X)<\infty$ porque $X$ é integrável por hipótese e $Y_{n}$ é $\mathcal{F}_{n}$ pela definição de esperança condicional. Vejamos agora que

$$
\mathbb{E}\left(Y_{n+1} \mid \mathcal{F}_{n}\right)=\mathbb{E}\left(\mathbb{E}\left(X \mid \mathcal{F}_{n+1}\right) \mid \mathcal{F}_{n}\right)=\mathbb{E}\left(X \mid \mathcal{F}_{n}\right)
$$

e usamos o ponto 7 da Proposição A.20.

Vejamos alguns exemplos de martingais. 
Exemplo 1. O mais básico exemplo é uma variável aleatória que assume sempre o mesmo valor, uma constante, i.e. $X_{n}=a$, para $a \in \mathbb{R} \forall n \in \mathbb{N}$ com um filtro $\left\{\mathcal{F}_{n} ; n \in \mathbb{N}\right\}$.

Exemplo 2. Considere a variável aleatória $X_{n}$ representando a $n$-ésima jogada de uma moeda justa. Se $X_{n}=1$ temos 'cara' como resultado e se $X_{n}=$ -1 temos 'coroa' como resultado da $n$-ésima jogada. Notemos que $\mathbb{E}\left(\left|X_{n}\right|\right)<\infty$ $\forall n \in \mathbb{N}$, ou seja, $X_{n}$ é uma variável aleatória integrável. Além disso, $\mathbb{E}\left(X_{n}\right)=0$ $\forall n \in \mathbb{N}$. Agora seja $S_{n}=X_{1}+X_{2}+\ldots+X_{n}$ e $\mathcal{F}_{n}=\sigma\left(X_{1}, X_{2}, \ldots X_{n}\right)$ a $\sigma$ álgebra gerada pelas variáveis aleatórias $X_{1}, X_{2}, \ldots X_{n}$ (ver A.5). Como $X_{0}=0$ e $\mathcal{F}_{0}=\{\emptyset, \Omega\}$. Vejamos que $\left\{S_{n}, \mathcal{F}_{n} ; n \in \mathbb{N}\right\}$ é um martingal.

Como as jogadas de moeda são independentes, $X_{n+1}$ não depende de resultados anteriores, i.e., não depende de $X_{1}, X_{2}, \ldots X_{n}$, logo $S_{n+1}$ é independente de $\mathcal{F}_{n}$. Vemos que $\mathbb{E}\left(\left|S_{n}\right|\right)<\infty$ e que $\mathbb{E}\left(S_{n}\right)=0$. Agora usando o ponto 4 e logo depois o ponto 3 da Proposição A.20 obtemos

$$
\begin{aligned}
\mathbb{E}\left(S_{n+1} \mid \mathcal{F}_{n}\right) & =\mathbb{E}\left(X_{n+1} \mid \mathcal{F}_{n}\right)+\mathbb{E}\left(S_{n} \mid \mathcal{F}_{n}\right) \\
& =\mathbb{E}\left(X_{n+1}\right)+S_{n} \\
& =S_{n}
\end{aligned}
$$

Por isso, concluímos que $\left\{S_{n}, \mathcal{F}_{n} ; n \in \mathbb{N}\right\}$ é um martingal.

Exemplo 3. Consideremos no espaço de probabilidade $(\Omega, \mathcal{F}, \mathbb{P})$ a sequência de variáveis aleatórias independentes $\left\{T_{n} ; n \in \mathbb{N}\right\}$ tal que $\forall n \in \mathbb{N}$ $\mathbb{E}\left(T_{n}\right)=0$ e $\mathbb{E}\left(\left|T_{n}\right|\right)<\infty$. Seja o filtro $\left\{\mathcal{F}_{n} ; n \in \mathbb{N}\right\}$ definido por $\mathcal{F}_{n}=$ $\sigma\left(T_{1}, T_{2}, \ldots, T_{n}\right)$ para cada $n \in \mathbb{N}$. Vejamos que

$$
X_{n}:=\prod_{i=1}^{n}\left(1+T_{i}\right)
$$

sob o filtro $\mathcal{F}_{n}$ é um martingal, i.e., $\left\{X_{n}, \mathcal{F}_{n} ; n \in \mathbb{N}\right\}$ é um martingal. Vejamos isso: Primeiro temos que verificar se $X_{n}$ é $\mathcal{F}_{n}$-mensurável. Note que $T_{1}, T_{2}, \ldots, T_{n}$ são $\mathcal{F}_{n}$-mensuráveis. A função $f: \mathbb{R}^{n} \longrightarrow \mathbb{R}$ definida por $f\left(x_{1}, x_{2}, \ldots, x_{n}\right)=\prod_{i=1}^{n}\left(1+x_{i}\right)$ é contínua, portanto é mensurável em borelianos de $\mathbb{R}^{n}$. Por esta razão, $X_{n}=f\left(T_{1}, T_{2}, \ldots, T_{n}\right)$ é $\mathcal{F}_{n}$-mensurável.

Vejamos agora a questão da integrabilidade. $\mathbb{E}\left(\left|X_{n}\right|\right)=\mathbb{E}\left(\left|\prod_{i=1}^{n}\left(1+T_{i}\right)\right|\right)$. Como $T_{1}, \ldots, T_{n}$ são independentes, pela desigualdade triangular e o fato de cada $T_{i}$ ser integrável teremos que $\mathbb{E}\left(\left|\prod_{i=1}^{n}\left(1+T_{i}\right)\right|\right)=\prod_{i=1}^{n} \mathbb{E}\left|\left(1+T_{i}\right)\right| \leqslant$ $\prod_{i=1}^{n}\left(1+\mathbb{E}\left|T_{i}\right|\right)<\infty$. 
Para a última propriedade, faremos

$$
\begin{aligned}
\mathbb{E}\left(X_{n+1} \mid \mathcal{F}_{n}\right) & =\mathbb{E}\left(\prod_{i=1}^{n+1}\left(1+T_{i}\right) \mid \mathcal{F}_{n}\right) \\
& =\prod_{i=1}^{n+1} \mathbb{E}\left(1+T_{i} \mid \mathcal{F}_{n}\right) \\
& =\prod_{i=1}^{n}\left(1+T_{i}\right) \mathbb{E}\left(1+T_{n+1} \mid \mathcal{F}_{n}\right)
\end{aligned}
$$

porque $\prod_{i=1}^{n}\left(1+T_{i}\right)=X_{n}$ é $\mathcal{F}_{n^{-}}$mensurável

$$
\begin{aligned}
& \left.=X_{n} \mathbb{E}\left(1+T_{n+1}\right) \mid \mathcal{F}_{n}\right) \\
& =X_{n}
\end{aligned}
$$

pelo fato de $\mathbb{E}\left(T_{n+1}\right)=0$. Com isso, concluímos que $\left\{X_{n}, \mathcal{F}_{n} ; n \in \mathbb{N}\right\}$ é um martingal.

Definição 3.9 Um processo $\left\{X_{n} ; n \in \mathbb{N}\right\}$ com filtro $\left\{\mathcal{F}_{n} ; n \in \mathbb{N}\right\}$ no espaço de probabilidade $\{\Omega, \mathcal{F}, \mathbb{P}\}$ que assume valores em $\mathbb{R}^{d}$ é dito um martingal quando cada coordenada é um martingal, i.e. $X_{n}=\left(X_{n}^{1}, X_{n}^{2}, \ldots X_{n}^{d}\right)$ é tal que $\left\{X_{n}^{j}, \mathcal{F}_{n} ; n \in \mathbb{N}\right\}$ é um martingal para cada $j \in\{1, \ldots, d\}$.

\section{3}

\section{Integração Estocástica Discreta}

Aqui, seguiremos o que foi definido no Apêndice A.13. A propriedade central da integração estocástica discreta que utilizaremos é que ao realizarmos transformações previsíveis(ver Apêndice A.12) às variações de um martingal, a cada instante de tempo, o novo processo conserva a propriedade de ser um martingal.

Proposição 3.10 Sejam $X_{n}$ e $H_{n}$ processos que tomam valores em $\mathbb{R}^{d} e$ definidos no mesmo espaço de probabilidade filtrado $(\Omega, \mathcal{F}, \mathbb{P}),\left\{\mathcal{F}_{n} ; n \in \mathbb{N}\right\}$. $S e\left\{X_{n}, \mathcal{F}_{n} ; n \in \mathbb{N}\right\}$ é um martingal e $H_{n}$ é previsivel e $\mathbb{E}\left(\left|H_{n}\right|\right)<\infty$, então o processo com valores em $\mathbb{R}, Y_{n}=\int_{0}^{n} H d X$ é um martingal.

Prova. Basta verificar que $\int_{0}^{n} H d X$ é um martingal. $Y_{n}$ é $\mathcal{F}_{n}$-mensurável, porque $Y_{n}=\sum_{i=1}^{n} H_{i} \cdot\left(X_{i}-X_{i-1}\right)$ é a soma de funções $f\left(H_{i}, X_{i}, X_{i-1}\right)=H_{i} \cdot\left(X_{i}-X_{i-1}\right)$ 
e cada função é $\mathcal{F}_{n}$-mensurável. Vejamos se $Y_{n}$ é integrável.

$$
\begin{aligned}
\mathbb{E}\left(\left|Y_{n}\right|\right)=\mathbb{E}\left(\left|\int_{0}^{n} H d X\right|\right) & =\mathbb{E}\left(\left|\sum_{i=1}^{n} H_{i} \cdot\left(X_{i}-X_{i-1}\right)\right|\right) \\
& \leqslant \sum_{i=1}^{n} \mathbb{E}\left(\left|H_{i} \cdot\left(X_{i}-X_{i-1}\right)\right|\right) \\
& <\infty .
\end{aligned}
$$

Onde usamos o fato de $H_{n}$ e $X_{n}$ serem integráveis. Agora, façamos

$$
\begin{aligned}
\mathbb{E}\left(\int_{0}^{n+1} H d X \mid \mathcal{F}_{n}\right) & =\mathbb{E}\left(\sum_{i=1}^{n+1} H_{i} \cdot\left(X_{i}-X_{i-1}\right) \mid \mathcal{F}_{n}\right) \\
& =\mathbb{E}\left(\int_{0}^{n} H d X+H_{n+1}\left(X_{n+1}-X_{n}\right) \mid \mathcal{F}_{n}\right) \\
& =\int_{0}^{n} H d X+H_{n+1} \mathbb{E}\left(X_{n+1}-X_{n} \mid \mathcal{F}_{n}\right) \\
& =\int_{0}^{n} H d X
\end{aligned}
$$

onde usamos a previsibilidade de $H_{n}$ na terceira igualdade e o fato de $\left\{X_{n}, \mathcal{F}_{n} ; n \in \mathbb{N}\right\}$ ser um martingal na última igualdade.

A integral estocástica discreta, como definida acima, possui as propriedades da linearidade da soma e produto por escalar real. Isso é fácil de ver, bastando tomarmos um processo previsível que é combinação de outros dois processos previsíveis $\left(a H_{n}+b Z_{n}\right)$, com $a, b \in \mathbb{R}$. Usaremos a integral estocástica como ferramenta em nosso modelo de mercado, sendo que o período final $N$ será nosso período final do modelo.

O próximo resultado será útil na sequência do texto. A partir de agora usaremos uma notação reduzida para o conjunto $\{1,2, \ldots N\}$, que será $\overline{1, N}$

Proposição 3.11 Seja $(\Omega, \mathcal{F}, \mathbb{P}) ;\left\{\mathcal{F}_{n} ; n \in \overline{1, N}\right\}$; um espaço de probabilidade filtrado e $\left\{X_{n} ; n \in \mathbb{N}\right\}$ um processo estocástico que assume valores em $\mathbb{R}^{d}$ com instante final $N .\left\{X_{n}, \mathcal{F}_{n} ; n \in \mathbb{N}\right\}$ é um martingal se e somente se para todo processo previsivel $H_{n}$ tomando valores em $\mathbb{R}^{d}$ temos que $\mathbb{E}\left(\int_{0}^{N} H d X\right)=0$.

Prova. Seja $\left\{X_{n}, \mathcal{F}_{n} ; n \in \overline{1, N}\right\}$ um martingal com valores em $\mathbb{R}^{d}$. Pela Proposição 3.10, $\int_{0}^{n} H d X$ é um martingal em $\mathbb{R}$. Usando a segunda propriedade da Proposição 3.7, vemos que $\mathbb{E}\left(\int_{0}^{n} H d X\right)=0$, pois pela Proposição A.13 o processo que define a integral estocástica é $\int_{0}^{n} H d X=Z_{n}-Z_{0}$.

Para a recíproca, vamos supor que $\mathbb{E}\left(\int_{0}^{n} H d X\right)=0$ para qualquer processo previsível $H_{n}$. Vamos mostrar que $X_{n}$ é um martingal fixando um instante $n_{0} \in\{1,2, \ldots N\}$. Por definição de esperança condicional, tomamos 
um conjunto $A \in \mathcal{F}_{0}$ arbitrário e mostraremos que

$$
\mathbb{E}\left[\left(X_{n_{0}+1}-X_{n_{0}}\right) \mathbb{I}_{A}\right]=0
$$

$\operatorname{com} \mathbb{I}_{A}$ sendo a função indicadora do conjunto $A$. Para isso criamos o processo previsível $H_{n}$ em que $H_{n}=0$ para $n \neq n_{0}+1$ e $H_{n_{0}+1}=\mathbb{I}_{A}$. Definindo $H_{n}$ desta forma, temos que

$$
\int_{0}^{N} H d X=\left(X_{n_{0}+1}-X_{n_{0}}\right) \mathbb{I}_{A}
$$

Então vemos que

$$
\mathbb{E}\left(\int_{0}^{n} H d X\right)=\mathbb{E}\left(X_{n_{0}+1}-X_{n_{0}} \mid \mathcal{F}_{n_{0}}\right)=0
$$

e assim, $\left\{X_{n} ; \mathcal{F}_{n} ; n \in \mathbb{N}\right\}$ é um martingal.

\section{4}

\section{O problema martingal}

Consideremos um espaço filtrado $(\Omega, \mathcal{F}) ;\left\{\mathcal{F}_{n} ; n \in \overline{1, N}\right\}$ e $\mathcal{F}=\mathcal{F}_{N}$. Manteremos sempre a hipótese de $|\Omega|<\infty$. O problema que avaliaremos aqui será o de, considerando $\left\{X_{n} ; n \in \overline{1, N}\right\}$ um processo estocástico com valores em $\mathbb{R}^{d}$ no espaço filtrado que definimos, saber se existe alguma medida de probabilidade $\mathbb{Q}$ que transforma $\left\{X_{n}, \mathcal{F}_{n} ; n \in \overline{1, N}\right\}$ em um martingal.

O problema acima é o nosso problema martingal. Definiremos $Q_{X}$ o conjunto de todas as medidas de probabilidade que são soluções do problema martingal.

Definição 3.12 Uma medida de probabilidade $\mathbb{Q}$ sobre o espaço mensurável $(\Omega, \mathcal{F})$ pertence a $Q_{X}$ se valem as seguintes condições:

- $\mathbb{Q}(w)>0 \forall w \in \Omega$.

- O processo $\left\{X_{n} ; n \in \overline{1, N}\right\}$ é um martingal assumindo valores em $\mathbb{R}^{d}$, com respeito a $\mathbb{Q}$, no espaço filtrado que fixamos no início.

O conjunto acima pode ser vazio ou ter vários elementos, ou seja, podemos não ter uma solução do problema, assim como poderemos ter uma ou várias.

Definimos o conjunto de variáveis aleatórias:

$$
\mathcal{A}(X):=\left\{\phi: \Omega \longrightarrow \mathbb{R} ; \phi=\int_{0}^{N} H d X ; \text { para algum processo } H_{n} \text { previsível }\right\} .
$$


Vemos que $\mathcal{A}(X)$ é um subespaço vetorial de $\mathbb{R}^{|\Omega|}$, graças a linearidade da integral estocástica. Cada variável aleatória $\phi$ também pode ser vista como um vetor em $\mathbb{R}^{|\Omega|}: \phi=\left(\phi\left(\omega_{1}\right), \phi\left(\omega_{2}\right), \ldots, \phi\left(\omega_{k}\right)\right)$ Desta forma, $\phi=0 \in \mathcal{A}(X) \mathrm{e}$ $\left(\phi_{1}+\alpha \phi_{2}\right) \in \mathcal{A}(X)$ com $\alpha \in \mathbb{R}$.

Para cada medida de probabilidade $\mathbb{Q}$ no espaço $(\Omega, \mathcal{F})$ definimos o conjunto

$$
\mathcal{B}(\mathbb{Q}):=\left\{\phi: \Omega \longrightarrow \mathbb{R} ; \mathbb{E}_{\mathbb{Q}}(\phi)=0\right\}
$$

Ou seja, $\mathcal{B}(\mathbb{Q})$ é o espaço das variáveis aleatórias com média zero em relação a $\mathbb{Q}$. O espaço $\mathcal{B}(\mathbb{Q})$ é um subespaço vetorial de $\mathbb{R}^{|\Omega|}$. Note que $\mathbb{Q}$ dá valores aos pontos $\left(\omega_{1}, \omega_{2}, \ldots \omega_{k}\right)$, portanto podemos ver os valores que $\mathbb{Q}$ toma como um vetor de $\mathbb{R}^{|\Omega|},\left(\mathbb{Q}\left(\omega_{1}\right), \mathbb{Q}\left(\omega_{2}\right), \ldots \mathbb{Q}\left(\omega_{k}\right)\right)$. Temos que o espaço $\mathcal{B}(\mathbb{Q})$ é o espaço onde $\mathbb{E}_{\mathbb{Q}}(\phi)=\phi \cdot \mathbb{Q}=0$, ou seja, é o espaço ortogonal a $\mathbb{Q}$.

Uma probabilidade $\mathbb{Q}$ em que $\mathbb{Q}(w)>0$ para todo $w \in \Omega$ é dita uma probabilidade não degenerada.

Lema 3.13 Uma probabilidade $\mathbb{Q}$ não degenerada pertence a $Q_{X}$ se e somente se $\mathcal{A}(X) \subseteq \mathcal{B}(\mathbb{Q})$, ou seja, $\mathbb{Q}$ é ortogonal a $\mathcal{A}(X)$.

Prova. Primeiro, notemos que $\mathbb{Q} \in Q_{X}$ e pela Definição 3.12 sabemos que $\left\{X_{n}, \mathcal{F}_{n} ; n \in \overline{1, N}\right\}$ é um martingal que toma valores em $\mathbb{R}^{d}$ com respeito à medida $\mathbb{Q}$. Graças à transformada martingal definida na Proposição A.13, sabemos que uma variável aleatória qualquer escrita como $\phi=\int_{0}^{N} H d X$ também pode ser escrita como um vetor que toma valores em $\mathbb{R}^{|\Omega|}$ para todo processo previsível $H_{n}$ e pela definição de $\mathcal{A}(X)$ temos que $\phi \in \mathcal{A}(X)$. Considerando a Proposição 3.11, temos que se $\left\{X_{n}, \mathcal{F}_{n} ; n \in \overline{1, N}\right\}$ é um martingal com valores em $\mathbb{R}^{d}$ então $\mathbb{E}_{\mathbb{Q}}\left(\int_{0}^{N} H d X\right)=\mathbb{E}_{\mathbb{Q}}(\phi)=0$, ou seja, qualquer variável $\int_{0}^{N} H d X$ que está em $\mathcal{A}(X)$ também estará em $\mathcal{B}(\mathbb{Q})$, portanto $\mathcal{A}(X) \subseteq \mathcal{B}(\mathbb{Q})$.

Seja uma variável aleatória $Y$ arbitrária em $\mathcal{A}(X) \subseteq \mathcal{B}(\mathbb{Q})$. Desta maneira $Y=\int_{0}^{N} H d X$ para algum $H_{n}$ previsível. Pela inclusão, $Y \in \mathbb{B}(\mathbb{Q}) \operatorname{logo}$ $\mathbb{E}_{\mathbb{Q}}(Y)=0$. Ou seja, $\mathbb{E}_{\mathbb{Q}}\left(\int_{0}^{N} H d X\right)=0$ para todo $H_{n}$ previsível. Agora pela Proposição 3.11 sabemos que um processo $\left\{X_{n}, n \in \overline{1, N}\right\}$ como o que compõe a integral acima é um martingal $\left\{X_{n}, \mathcal{F}_{n} ; n \in \overline{1, N}\right\}$ no espaço $(\Omega, \mathcal{F}, \mathbb{Q})$. Concluímos que se $\mathbb{Q}$ é não degenerada, então é solução do problema martingal, i.e., $\mathbb{Q} \in Q_{X}$.

As seguintes proposições são importantes e relacionam a unicidade de uma solução do problema martingal com integrais que representam martingais de média zero. O sentido destas demonstrações virá quando representarmos coberturas e processos de preços de contratos usando integrais e martingais. 
Usaremos estas proposições nas demonstrações dos teoremas fundamentais deste capítulo.

Proposição 3.14 Suponhamos que existe uma única solução do problema martingal, ou seja, $Q_{X}=\{\mathbb{Q}\}$. Então todo martingal $\left\{Z_{n}, \mathcal{F}_{n} ; n \in \overline{1, N}\right\}$ assumindo valores em $\mathbb{R}$ e com média zero com relação à medida $\mathbb{Q}$ é da forma $Z_{n}=\int_{0}^{n} H d X$ para $n \in \overline{0, N}$ e para algum processo previsivel $H_{n}$ que toma valores em $\mathbb{R}^{d}$.

Prova. Vamos provar por contradição. Já sabemos pelo lema acima que se $\mathbb{Q} \in Q_{X}$ então $\mathcal{A}(X) \subseteq \mathcal{B}(\mathbb{Q})$. Suponha que existe $Z_{N} \in \mathcal{B}(\mathbb{Q})$, mas $Z_{N} \notin \mathcal{A}(X)$. Dessa forma teríamos $\mathcal{A}(X) \subsetneq \mathcal{B}(\mathbb{Q})$. Então existe algum vetor $q \in \mathcal{B}(\mathbb{Q}) \backslash \mathcal{A}(X)$. Por álgebra linear, sabemos que $\mathbb{R}^{d}=\mathcal{A}(X) \oplus \mathcal{A}(X)^{\perp}$. Então $q \in \mathcal{A}(X)^{\perp} \cap \mathcal{B}(\mathbb{Q})$.

Sendo $\mathbb{Q} \in Q_{X}$ vale $\mathbb{Q}(\omega)>0 \forall w \in \Omega$. Existe $\delta>0$ tão pequeno quanto desejamos tal que sempre temos $\mathbb{Q}(\omega)+\delta . q(\omega)>0$ Podemos então construir

$$
\mathbb{Q}^{\prime}(\omega):=s(\mathbb{Q}(\omega)+\delta . q(\omega))
$$

onde $s=\sum_{\omega \in \Omega} \frac{1}{[\mathbb{Q}(\omega)+\delta . q(\omega)]}$ é uma constante de normalização. Notemos que $\mathbb{Q}^{\prime}$ assim construída é uma medida de probabilidade, pois $\mathbb{Q}^{\prime}(w) \in(0,1)$ e $\sum_{\omega_{i} \in A} \mathbb{Q}^{\prime}\left(\omega_{i}\right)=\mathbb{Q}^{\prime}\left(\cup_{\omega_{i} \in A}\left\{\omega_{i}\right\}\right)$ para qualquer subconjunto $A$ de elementos de $\Omega$. Por definição, $\mathbb{Q}^{\prime}(w)>0 \forall w \in \Omega$. Como $q \in \mathcal{B}(\mathbb{Q})$ sabemos que $q$ é ortogonal à $\mathbb{Q}$ e com isso $\mathbb{Q}^{\prime} \neq \mathbb{Q}$.

Agora vamos mostrar que $\mathbb{Q}^{\prime}$ como geramos acima é solução do problema martingal. Para isso, vamos mostrar que $\mathcal{A}(X) \subseteq B\left(\mathbb{Q}^{\prime}\right)$ e o resultado do Lema 3.13. Seja $\phi \in \mathcal{A}(X)$. Então

$$
\mathbb{E}_{\mathbb{Q}^{\prime}}(\phi)=\sum_{w \in \Omega} \phi(w) \mathbb{Q}^{\prime}(w)=s . \mathbb{E}_{\mathbb{Q}}(\phi)+s . \delta \sum_{w \in \Omega} \phi(w) . q(w)=0 .
$$

Acima usamos o fato que $\mathbb{Q}$ é solução do problema martingal e que $q$ é ortogonal a $\phi$.

Mostramos que $\mathbb{E}_{\mathbb{Q}^{\prime}}(\phi)=0$ e que $\mathcal{A}(X) \subseteq B\left(\mathbb{Q}^{\prime}\right)$. Temos $\mathbb{Q}^{\prime}$ diferente de $\mathbb{Q}$ solução do problema martingal pelo Lema 3.13, ou seja, um absurdo. Assim concluímos a prova.

Proposição 3.15 Suponhamos que $Q_{X} \neq \emptyset$ e tome $\mathbb{Q} \in Q_{X}$. Se todo martingal assumindo valores em $\mathbb{R}$ de média zero com respeito a $\mathbb{Q}$ é da forma $\int_{0}^{n} H d X$ então $\mathbb{Q}$ é a única solução do problema martingal.

Prova. Sejam $\mathbb{Q}$ e $\mathbb{Q}^{\prime} \in Q_{X}$ duas soluções do problema martingal. Fixemos um conjunto arbitrário $A \in \mathcal{F}$ e mostraremos que $\mathbb{Q}(A)=\mathbb{Q}^{\prime}(A)$, ou seja, as 
medidas $\mathbb{Q}^{\prime}$ e $\mathbb{Q}$ são iguais. Defina a seguinte variável aleatória

$$
Z_{n}:=\mathbb{E}_{\mathbb{Q}}\left[\mathbb{I}_{A}-\mathbb{Q}(A) \mid \mathcal{F}_{n}\right]
$$

Vejamos que $\left\{Z_{n}, \mathcal{F}_{n} ; n \in \mathbb{N}\right\}$ é um martingal de média zero. Do item 1 da Definição A.19 vemos que $Z_{n}$ é $\mathcal{F}_{n}$-mensurável. Agora note que

$$
\mathbb{E}_{\mathbb{Q}}\left(\left|Z_{n}\right|\right)=\mathbb{E}_{\mathbb{Q}}\left(\left|\mathbb{E}_{\mathbb{Q}}\left[\mathbb{I}_{A}-\mathbb{Q}(A) \mid \mathcal{F}_{n}\right]\right|\right), \text { e }
$$

usando a Desigualdade de Jensen (ponto 10 da Proposição A.20) teremos

$$
\mathbb{E}_{\mathbb{Q}}\left(\left|\mathbb{E}_{\mathbb{Q}}\left[\mathbb{I}_{A}-\mathbb{Q}(A) \mid \mathcal{F}_{n}\right]\right|\right) \leqslant \mathbb{E}_{\mathbb{Q}}\left(\mathbb{E}_{\mathbb{Q}}\left[\left|\mathbb{I}_{A}-\mathbb{Q}(A)\right| \mid \mathcal{F}_{n}\right]\right)
$$

Usando o ponto 9 da Proposição A.20 teremos que

$$
\mathbb{E}_{\mathbb{Q}}\left(\mathbb{E}_{\mathbb{Q}}\left[\left|\mathbb{I}_{A}-\mathbb{Q}(A)\right| \mid \mathcal{F}_{n}\right]\right)=\mathbb{E}_{\mathbb{Q}}\left[\left|\mathbb{I}_{A}-\mathbb{Q}(A)\right|\right] \leqslant 2 \mathbb{Q}(A)
$$

Com isso $\mathbb{E}_{\mathbb{Q}}\left(\left|Z_{n}\right|\right) \leqslant 2 \mathbb{Q}(A)$ e temos que $Z_{n}$ é integrável. Agora devemos mostrar que $Z_{n}$ tem a propriedade martingal. Ora

$$
\mathbb{E}_{\mathbb{Q}}\left(Z_{n+1} \mid \mathcal{F}_{n}\right)=\mathbb{E}_{\mathbb{Q}}\left(\mathbb{E}_{\mathbb{Q}}\left[\mathbb{I}_{A}-\mathbb{Q}(A) \mid \mathcal{F}_{n+1}\right] \mid \mathcal{F}_{n}\right)
$$

e pelo ponto 7 da Proposição A.20 temos que

$$
\mathbb{E}_{\mathbb{Q}}\left(\mathbb{E}_{\mathbb{Q}}\left[\mathbb{I}_{A}-\mathbb{Q}(A) \mid \mathcal{F}_{n+1}\right] \mid \mathcal{F}_{n}\right)=\mathbb{E}_{\mathbb{Q}}\left[\mathbb{I}_{A}-\mathbb{Q}(A) \mid \mathcal{F}_{n}\right]=Z_{n}
$$

Logo $\mathbb{E}_{\mathbb{Q}}\left(Z_{n+1} \mid \mathcal{F}_{n}\right)=Z_{n}$. Daqui resulta $\left\{Z_{n} ; n \in \overline{1, N}\right\}$ é um martingal.

Além disso, $Z_{n}$ tem média zero porque $\mathbb{E}_{\mathbb{Q}}\left(Z_{n}\right)=\mathbb{E}_{\mathbb{Q}}\left(\mathbb{I}_{A}-\mathbb{Q}(A)\right)=$ $\mathbb{Q}(A)-\mathbb{Q}(A)=0$.

Por hipótese, existe $H_{n}$ previsível tal que $Z_{n}=\int_{0}^{n} H d X$. Sabemos que $\left\{X_{n}, \mathcal{F}_{n} ; n \in \mathcal{F}_{n}\right\}$ é um martingal com valores em $\mathbb{R}^{d}$ com relação a $\mathbb{Q}^{\prime}, \operatorname{logo}\left\{Z_{n}, \mathcal{F}_{n} ; n \in \mathbb{N}\right\}$ é um martingal com respeito a $\mathbb{Q}^{\prime}$. Pela segunda propriedade da Proposição 3.7 e sabendo que $Z_{0}=0$, temos $\forall n \in \overline{1, N}$, $\mathbb{E}_{\mathbb{Q}^{\prime}}\left(Z_{n}\right)=\mathbb{E}_{\mathbb{Q}^{\prime}}\left(Z_{0}\right)=0$. Daí $\mathbb{E}_{\mathbb{Q}^{\prime}}\left(Z_{N}\right)=0$.

Como $\mathcal{F}=\mathcal{F}_{N}$ e $A$ é $\mathcal{F}$-mensurável, teremos $Z_{N}=\mathbb{I}_{A}-\mathbb{Q}(A)$ q.c. Usando isto em $\mathbb{E}_{\mathbb{Q}^{\prime}}\left(Z_{N}\right)$ temos que

$$
\begin{aligned}
0=\mathbb{E}_{\mathbb{Q}^{\prime}}\left(Z_{N}\right) & =\mathbb{E}_{\mathbb{Q}^{\prime}}\left[\mathbb{I}_{A}-\mathbb{Q}(A)\right] \\
& =\mathbb{E}_{\mathbb{Q}^{\prime}}\left(\mathbb{I}_{A}\right)-\mathbb{Q}(A) \\
& =\mathbb{Q}^{\prime}(A)-\mathbb{Q}(A) .
\end{aligned}
$$


Portanto $\mathbb{Q}^{\prime}(A)=\mathbb{Q}(A) \quad \forall A \in \mathcal{F}$ o que implica que $\mathbb{Q}^{\prime}=\mathbb{Q}$ Assim, a solução do problema martingal é única.

\section{5}

\section{Teoremas Fundamentais}

A partir deste ponto vamos tratar diretamente com o mercado a vários períodos de tempo, tentando modelá-lo com as ferramentas que foram apresentadas até aqui. Mostraremos os dois teoremas fundamentais que nos permitirão determinar sob que condições poderemos determinar o preço justo de contratos.

Definição 3.16 Seja o mercado $\left\{M_{n} ; n \in \overline{1, N}\right\}$ um processo estocástico que toma valores em $\mathbb{R}^{\ell+1}$. Associamos a este mercado o processo

$$
\overline{M_{n}}=\frac{M_{n}}{B_{n}}
$$

que chamamos de processo de preços descontados.

O ativo pelo qual dividimos $M_{n}$, neste caso $B_{n}$, é conhecido como numerário. Notemos que o preço do primeiro ativo em $\overline{M_{n}}$ é sempre igual a 1, daí se tratar de um mercado onde a taxa de juros é nula.

Proposição 3.17 Seja o mercado $M_{n}$. Tome como numerário o ativo $S_{n}^{i}$. Uma estratégia $\Gamma_{n}$ é dita auto-financiada com relação a $M_{n}$ se e somente se é auto-financiada com relação a $\overline{M_{n}}$.

Prova. É claro que supomos que todos os preços são positivos. Como $\Gamma_{n}$ é autofinanciada (ver Proposição 2.19) $\Gamma_{n} \cdot M_{n}=\Gamma_{n+1} \cdot M_{n} \forall n \in \overline{1, N}$. Dividindo ambos os lados da igualdade anterior por $S_{n}^{i}$, obteremos $\Gamma_{n} \cdot \overline{M_{n}}=\Gamma_{n+1} \cdot \overline{M_{n}}$. Portanto a estratégia é auto-financiada com relação a $\overline{M_{n}}$.

Por uma razão semelhante ao que afirmamos acima, podemos ver que o mercado $\overline{M_{n}}$ é livre de arbitragem se e somente se $M_{n}$ o é.

Adotaremos uma notação reduzida a partir de agora. Façamos $\overline{S_{n}^{i}}=\frac{S_{n}^{i}}{B_{n}}$ para $i \in\{1,2, \ldots \ell\}$ tal que $\overline{M_{n}}=\left(1, \overline{S_{n}^{1}}, \ldots \overline{S_{n}^{\ell}}\right)$. Com $\overline{S_{n}}=\left(\overline{S_{n}^{1}}, \ldots \overline{S_{n}^{\ell}}\right)$, escreveremos $\overline{M_{n}}=\left(1, \overline{S_{n}}\right)$. Se assumirmmos que a taxa de juros seja sempre nula, então temos $M_{n}=\left(1, S_{n}\right)=\left(1, \overline{S_{n}}\right)$.

O problema martingal que tratamos anteriormente pode ser adaptado para este contexto como $Q_{\bar{S}}$, ou seja, o conjunto das medidas de probabilidade que são solução do problema martingal para o processo estocástico $\left\{S_{n} ; n \in\right.$ $\overline{0, N}\}$ no espaço filtrado $(\Omega, \mathcal{F}, \mathbb{Q}),\left\{\mathcal{F}_{n} ; n \in \overline{0, N}\right\}$. 
Definição 3.18 Cada probabilidade em $Q_{\bar{S}}$ é dita probabilidade neutra ao risco para o mercado $M_{n}$.

Lema 3.19 Seja $\alpha_{n}$ um processo assumindo valores em $\mathbb{R}^{\ell}$ e previsível. Seja $c \in \mathbb{R}$. Existe sempre um portfólio $\Gamma_{n}$ auto-financiado tal que $\Gamma_{n}=$ $\left(\beta_{n}, \alpha_{n}\right), \quad \forall n \in\{1,2, \ldots N\}$ e $V_{0}^{\Gamma}=c$.

A prova do Lema anterior pode ser encontrada em, [2] por exemplo.

Lema 3.20 Seja $\mathbb{Q} \in Q_{\bar{S}}$ e $\Gamma_{n}$ auto-financiado. Então $V_{n}^{\Gamma}$ é um martingal que toma valores em $\mathbb{R}$ com respeito a $\mathbb{Q}$.

Prova. Pela Proposição 2.21 sabemos que $\Gamma_{n}$ é auto-financiada se e somente se $V_{n}^{\Gamma}=V_{0}^{\Gamma}+\int_{0}^{n} \Gamma d M$. Como $\Gamma_{n}=\left(\beta_{n}, \alpha_{n}\right)$ e $\Delta M_{n}=\left(0, \Delta S_{n}\right)$ vemos que $\int_{0}^{n} \Gamma d M=\int_{0}^{n} \alpha d S$.

Como $\mathbb{Q} \in Q_{\bar{S}}$ temos que $\left\{S_{n}, \mathcal{F}_{n} ; n \in \overline{1, N}\right\}$ é um martingal que toma valores em $\mathbb{R}^{d}$. Portanto $V_{n}^{\Gamma}$ é um martingal com respeito a $\mathbb{Q}$.

Consideremos o conjunto de variáveis aleatórias

$$
C=\left\{\xi: \Omega \longrightarrow \mathbb{R} ; \xi(\omega) \geqslant 0 \forall \omega \in \Omega, \sum_{w \in \Omega} \xi(w)=1\right\}
$$

O conjunto $C$ é convexo e isso pode ser visto sem dificuldades. Este conjunto é compacto em $\mathbb{R}^{|\Omega|}$ e para isso devemos verificar que é limitado e fechado em $\mathbb{R}^{|\Omega|}$. $C$ é limitado pela bola usual em $\mathbb{R}^{|\Omega|}$, ou seja, pelo conjunto de pontos $B_{1}\left(\left(x_{1}, x_{2}, \ldots, x_{|\Omega|}\right):\left|\left(x_{1}, x_{2}, \ldots, x_{|\Omega|}\right)\right| \leq 1\right)$ onde $|$.$| é a distância euclideana.$ Considere uma sequência $\left\{\xi_{n} ; n \in \mathbb{N}\right\}$ de variáveis aleatórias de $C$ convergindo à $\xi$ com respeito a métrica euclideana. Como o limite de uma sequência de variáveis aleatórias é uma variável aleatória, sabemos que $\xi$ é variável aleatória. Agora, $\lim _{n \rightarrow \infty} \xi_{n}(\omega)=\xi(\omega), \log \mathrm{O}$

$$
\sum_{\omega \in \Omega} \xi(\omega)=\sum_{\omega \in \Omega} \lim \xi_{n}(\omega)
$$

e por $\Omega$ ser finito teremos

$$
\sum_{\omega \in \Omega} \lim \xi_{n}(\omega)=\lim \sum_{\omega \in \Omega} \xi_{n}(\omega)=1
$$

o que implica que $\sum_{\omega \in \Omega} \xi(\omega)=1$. Logo $\xi \in C$ e o conjunto é compacto e limitado, como queríamos mostrar.

Usaremos novamente o conjunto das variáveis aleatórias dado por

$$
\mathcal{A}(S)=\left\{\phi: \Omega \longrightarrow \mathbb{R} ; \phi=\int_{0}^{N} \alpha d S \text { para algum } \alpha_{n} \text { previsível. }\right\}
$$


Lema 3.21 $M_{n}$ é livre de arbitragem se e somente se $\mathcal{A}(S) \cap C=\emptyset$.

Prova. Vamos provar a primeira implicação por contradição. Suponha que $\exists \xi \in \mathcal{A}(S) \cap C$. Então $\exists \alpha_{n}$ previsível tal que $\xi=\int_{0}^{N} \alpha d S \geqslant 0$ e $\xi\left(w_{0}\right)>0$ para algum $w_{0} \in \Omega$.

Aplicamos o Lema 3.19 para $c=0$. Sabemos que temos um portfólio auto-financiado $\Gamma_{n}=\left(\beta_{n}, \alpha_{n}\right)$. Pela Proposição 2.21 e como $V_{0}^{\Gamma}=0$ temos que $V_{N}^{\Gamma}=\int_{0}^{N} \alpha d S$. Disto implica que $V_{0}^{\Gamma}=\xi=\int_{0}^{N} \alpha d S \geqslant 0$ e $V_{0}^{N}\left(w_{0}\right)=\xi\left(w_{0}\right)=$ $\int_{0}^{N} \alpha d S>0$. Então haverá alguma oportunidade de arbitragem, o que é um absurdo.

Agora para provar a outra implicação, notemos que se existe uma oportunidade de arbitragem, ou seja, uma estratégia $\Gamma_{n}=\left(\beta_{n}, \alpha_{n}\right)$ capaz de gerar arbitragem, temos $V_{N}^{\Gamma}=\int_{0}^{N} \alpha d S \geqslant 0$. Se multiplicarmos $V_{N}^{\Gamma}$ por $\left(\sum_{w \in \Omega} \int_{0}^{N} \alpha d S(w)\right)^{-1}$ estaremos normalizando $\int_{0}^{N} \alpha d S$ de maneira que a variável aleatória gerada será maior igual que zero e terá soma igual a 1 e sendo escrita por uma integral. Por isso teremos esta nova variável aleatória em $\mathcal{A}(S) \cap C$ e concluímos a prova.

O próximo Teorema relaciona as estratégias que geram arbitragem com a existência de medidas de probabilidade neutras ao risco. Um mercado não irá admitir oportunidades de arbitragem se existe alguma medida de probabilidade neutra ao risco. Intuitivamente é um resultado que faz sentido, já que se há uma probabilidade neutra ao risco sabemos que é possível calcular o preço correto de um ativo no mercado, eliminando assim a má precificação que pode gerar ganhos por arbitragem, como vimos em alguns exemplos no Capítulo 2.

Teorema $3.22 M_{n}$ é livre de arbitragem se e somente se $Q_{\bar{S}} \neq \emptyset$.

Prova. Sabemos que o conjunto $\mathcal{A}(S)$ é um subespaço vetorial de $\mathbb{R}^{|\Omega|}$ e também sabemos que $C$ é convexo e compacto, logo também fechado. Usando uma versão do Teorema de Hahn-Banach conhecido como Teorema do Hiperplano Separador (veja [2] e [3]) existe um vetor $q \in \mathbb{R}^{|\Omega|}$ ortogonal a $\mathcal{A}(S)$ tal que $q \cdot \xi>0 \forall \xi \in C$. Os vetores canônicos $E=\left\{e_{1}, e_{2}, \ldots e_{k}\right\} \in C$, logo $\left(q\left(w_{1}\right), q\left(w_{2}\right), \ldots q\left(w_{k}\right)\right) \cdot(0, \ldots 0,1,0 \ldots 0)=q\left(w_{i}\right)>0$. Temos então $q(w)>0$ $\forall \omega \in \Omega$. Sabendo que $q>0$ podemos normalizar o vetor de maneira que se torne uma probabilidade $\mathbb{Q}$.

Façamos

$$
\mathbb{Q}\left(\omega_{i}\right)=\frac{q\left(\omega_{i}\right)}{\sum_{\omega \in \Omega} q(\omega)}>0, \forall \omega_{i} \in \Omega
$$


Como $\mathbb{Q}$ é múltipla de $q$ então é ortogonal a $\mathcal{A}(S)$. Pelo Lema 3.13 concluímos que $\mathbb{Q} \in Q_{\bar{S}}$.

Reciprocamente, se existe $\mathbb{Q} \in Q_{\bar{S}}$ então pelo Lema 3.13 temos que $\mathcal{A}(S) \subseteq B(\mathbb{Q})$. Logo, $\mathbb{E}_{\mathbb{Q}}(\xi)=0 \quad \forall \xi \in \mathcal{A}(S)$, ou seja, $\mathbb{Q} \cdot \xi=0$. É claro que $\xi \notin C$, pois $\mathbb{Q} \cdot \xi=0 \Longrightarrow \xi=0$ o que torna impossível $\sum_{w \in \Omega} \xi(w)=1$. Portanto pelo Lema 3.21, concluímos que $M_{n}$ é livre de arbitragem.

O lema seguinte irá auxiliar no teorema que mostraremos em sequência. O teorema irá relacionar a existência de medidas de probabilidade neutra ao risco com a completude do mercado. Com a existência de mais de uma medida de probabilidade neutra ao risco, ativos de risco como os contratos nem sempre terão o mesmo preço correto. Ou seja, pode haver mais de uma forma de cobrir algum contrato no mercado.

Lema 3.23 Sejam $X_{n}$ e $Y_{n}$ dois martingais sob o mesmo espaço filtrado $(\Omega, \mathcal{F}, \mathbb{P}),\left\{\mathcal{F}_{n} ; n \in \overline{0, N}\right\}$. Se $X_{N}=Y_{N}$ então $X_{n}=Y_{n}$ q.c. $\forall n \in \mathbb{N}$.

Prova. Seja $M_{n}=X_{n}-Y_{n}$ um processo criado a partir dos martingais do enunciado. Como $X_{N}=Y_{N}$ então $M_{N}=0$. Vemos que $\mathbb{E}\left(M_{N} \mid \mathcal{F}_{N-1}\right)=$ $X_{N-1}-Y_{N-1}=M_{N-1}$ pois as duas variáveis são martingais. Fica claro que $M_{n}$ é um martingal no mesmo filtro e mesma medida de probabilidade do que suas variáveis constituintes. Pelo ponto 2 da Proposição 3.7 sabemos que $\mathbb{E}\left(M_{k} \mid \mathcal{F}_{n}\right)=X_{n}-Y_{n} \forall k>n$. Pelo ponto 1 da mesma Proposição sabemos que a média de um martingal é constante, por isso $\mathbb{E}\left(M_{N}\right)=\mathbb{E}\left(M_{n}\right)=0$ e temos $X_{n}=Y_{n}$ q.c., como queríamos demonstrar.

Teorema 3.24 Suponhamos $M_{n}$ livre de arbitragem. $M_{n}$ é completo se e somente se existe uma única solução em $Q_{\bar{S}}$.

Prova. Neste Teorema iremos considerar o caso em que $r=0$ para facilidade de notação. Primeiro vamos provar que se há uma única solução do problema martingal, então $M_{n}$ é completo. Se $M_{n}$ é livre de arbitragem, sabemos pelo teorema anterior que há alguma solução ao problema martingal. Vamos mostrar que se $\mathbb{Q}$ é a única solução do problema martingal, $\mathbb{Q} \in Q_{X}$ então o mercado $M_{n}$ é completo. Considere $F$ um contrato arbitrário e um filtro $\left\{\mathcal{F}_{n} ; n \in \overline{0, N}\right\}$. Seja o processo

$$
F_{n}:=\mathbb{E}_{\mathbb{Q}}\left(F_{N} \mid \mathcal{F}_{n}\right)-\mathbb{E}_{\mathbb{Q}}\left(F_{N}\right), \quad n \in \overline{0, N}
$$

Este processo tem média zero. Para tal, aplicando a esperança, temos

$$
\mathbb{E}_{\mathbb{Q}}\left(F_{n}\right)=\mathbb{E}_{\mathbb{Q}}\left(\mathbb{E}_{\mathbb{Q}}\left(F_{N} \mid \mathcal{F}_{n}\right)-\mathbb{E}_{\mathbb{Q}}\left(F_{N}\right)\right)=\mathbb{E}_{\mathbb{Q}}\left(F_{N}\right)-\mathbb{E}_{\mathbb{Q}}\left(F_{N}\right)=0
$$


Acima usamos o ponto 9 da Proposição A.20 ao passar da primeira para a segunda igualdade. Se fizermos $\mathbb{E}_{\mathbb{Q}}\left(F_{n+1} \mid \mathcal{F}_{n}\right)$ teremos

$$
\mathbb{E}_{\mathbb{Q}}\left(F_{n+1} \mid \mathcal{F}_{n}\right)=\mathbb{E}_{\mathbb{Q}}\left(\mathbb{E}_{\mathbb{Q}}\left(F_{N} \mid \mathcal{F}_{n+1}\right)-\mathbb{E}_{\mathbb{Q}}\left(F_{N}\right) \mid \mathcal{F}_{n}\right)=F_{n}
$$

que é uma das propriedades exigidas para que $\left\{F_{n}, \mathcal{F}_{n} ; n \in \overline{0, N}\right\}$ seja um martingal. $F_{n}$ é $\mathcal{F}_{n}$-mensurável, pois é formado por uma termo que é uma esperança condicional $\left(\mathcal{F}_{n}\right.$-mensurável por definição) e $\mathbb{E}_{\mathbb{Q}}\left(F_{N}\right)$ que é um valor real. Vejamos se $F_{n}$ é integrável. Ora

$$
\begin{aligned}
\mathbb{E}_{\mathbb{Q}}\left(\left|F_{n}\right|\right) & =\mathbb{E}_{\mathbb{Q}}\left(\left|\mathbb{E}_{\mathbb{Q}}\left(F_{N} \mid \mathcal{F}_{n}\right)-\mathbb{E}_{\mathbb{Q}}\left(F_{N}\right)\right|\right) \\
& =\mathbb{E}_{\mathbb{Q}}\left(\left|\mathbb{E}_{\mathbb{Q}}\left(F_{N}-\mathbb{E}_{\mathbb{Q}}\left(F_{n}\right) \mid \mathcal{F}_{n}\right)\right|\right) \\
& \leqslant \mathbb{E}_{\mathbb{Q}}\left(\mathbb{E}_{\mathbb{Q}}\left(\left|F_{N}-\mathbb{E}_{\mathbb{Q}}\left(F_{N}\right)\right| \mid \mathcal{F}_{n}\right)\right) \\
& =\mathbb{E}_{\mathbb{Q}}\left(\left|F_{N}-\mathbb{E}_{\mathbb{Q}}\left(F_{N}\right)\right|\right) \\
& \leqslant 2 \mathbb{E}_{\mathbb{Q}}\left(F_{N}\right)=0 .
\end{aligned}
$$

Acima usamos os pontos 10 e 9 da Proposição A.20 na segunda igualdade e para obter a desigualdade, respectivamente. Por fim, usamos o fato do processo ter média zero. Pelas três propriedades que mostramos, sabemos que $\left\{F_{n}, \mathcal{F}_{n} ; n \in \overline{0, N}\right\}$ é um martingal.

Pela Proposição 3.14, sabemos que qualquer martingal de média zero pode ser escrito na forma $F_{n}=\int_{0}^{n} \alpha d S$ para $\alpha_{n}$ um processo previsível que toma valores em $\mathbb{R}^{\ell}$. Usando o Lema 3.19, já sabemos que $\alpha_{n}$ é previsível e tomaremos $c=\mathbb{E}_{\mathbb{Q}}\left(\overline{F_{N}}\right)$. Assim temos que

$$
F_{n}=\int_{0}^{n} \alpha d S \text { e } \int_{0}^{n} \alpha d S=\mathbb{E}_{\mathbb{Q}}\left(F_{N} \mid \mathcal{F}_{n}\right)-c
$$

onde substituímos $F_{n}$ pelo nosso martingal definido no início da demonstração. Pelo mesmo Lema 3.19 sabemos que existe $\Gamma_{n}=\left(\alpha_{n}, \beta_{n}\right)$ auto-financiado tal que $V_{0}^{\Gamma}=\mathbb{E}_{\mathbb{Q}}\left(F_{N}\right)$. Relembrando a Proposição 2.21, vemos que $V_{n}^{\Gamma}=$ $\mathbb{E}_{\mathbb{Q}}\left(F_{N} \mid \mathcal{F}_{n}\right), \forall n \in \overline{0, N}$. Por isso,

$$
V_{N}^{\Gamma}=\mathbb{E}_{\mathbb{Q}}\left(F_{N} \mid \mathcal{F}_{N}\right)=F_{N}
$$

e provamos que $\Gamma_{n}$ é uma cobertura de $F_{n}$, logo o mercado é completo.

Vamos provar a recíproca. Se $M_{n}$ é completo então $\mathbb{Q}$ é a única solução do problema martingal. Considere um contrato $Z_{N}: \Omega \rightarrow \mathbb{R}$ e seja $\left\{Z_{n}, \mathcal{F} ; n \in\right.$ $\overline{0, N\}}$ um martingal que toma valores em $\mathbb{R}$ com média zero com respeito a $\mathbb{Q}$, que existe, pois sabemos que $M_{n}$ é livre de arbitragem e possui uma solução do problema martingal (o fato de ter média zero sabemos do ponto 1 
do Lema 3.8). Como $M_{n}$ é completo, existe $\Gamma_{n}$ auto-financiado que é cobertura de $Z_{N}$. Sabemos que $\mathbb{Q} \in Q_{\bar{S}}$ é probabilidade neutra ao risco e pelo Lema 3.20 temos que $\left\{V_{n}^{\Gamma}, \mathcal{F}_{n} ; n \in \overline{0, N}\right\}$ é um martingal com valores em $\mathbb{R}$. Como $V_{N}^{\Gamma}=Z_{N}$ e $\Gamma_{n}$ é cobertura de $Z$, devemos ter $Z_{n}=V_{n}^{\Gamma}, \quad 0 \leqslant n \leqslant N$ (pelo Lema 3.23). Com isso,

$$
Z_{n}=V_{n}^{\Gamma}=\int_{0}^{n} \alpha d S
$$

Provamos que qualquer martingal de média zero em relação a $\mathbb{Q}$ é escrito da forma $\int_{0}^{n} H d S$ para $H$ previsível e pela Proposição 3.15 sabemos que $\mathbb{Q}$ é solução única do problema martingal.

Lema 3.25 Se $M_{n}$ é livre de arbitragem, $\mathbb{Q}$ é uma probabilidade neutra ao risco e $\Gamma_{n}$ é uma estratégia auto-financiada então $\overline{V_{n}^{\Gamma}}=\Gamma \cdot \overline{M_{n}}$ é um martingal com respeito à $\mathbb{Q}$.

Prova. Como estamos considerando $M_{n}=\left(1, S_{n}\right)$ e $\overline{S_{n}}=S_{n}$ então $M_{n}=\overline{M_{n}}$. Então agora a prova é idêntica ao caso $M_{n}$. (Nota: Também podemos considerar $\overline{V_{n}^{\Gamma}}$ um martingal pelo fato de ser uma transformada martingal).

Na seguinte proposição mostrará que para calcularmos o preço correto de um contrato não é necessário que saibamos explicitamente qual sua cobertura. Sabendo que existe uma cobertura já é uma informação valiosa e considerando a probabilidade-nos calcular o único preço correto do contrato $F$.

Proposição 3.26 Seja $M_{n}$ um mercado livre de arbitragem e $F: \Omega \longrightarrow \mathbb{R}$ um contrato. Se F possui um portfólio de cobertura $\Gamma_{n}$, então $F$ tem um único processo de preços corretos $\left\{P_{n}(F) ; n \in \overline{0, N}\right\}$. Além disso, $P_{n}(F)=V_{n}^{\Gamma}, \forall n \in$ $\overline{0, N}$ e se $\mathbb{Q}$ é uma probabilidade neutra ao risco então

$$
P_{n}(F)=B_{n} \mathbb{E}_{\mathbb{Q}}\left(\frac{F}{B_{N}} \mid \mathcal{F}_{n}\right)
$$

Em particular, $P_{0}(F)=B_{0} \mathbb{E}_{\mathbb{Q}}\left(B_{N}^{-1} F\right)$.

Prova. Seja $P_{n}(F)$ o processo de preços corretos de $F$ e $\Gamma_{n}$ a cobertura do contrato. Pela Definição 2.22 sabemos que o mercado $M_{n}^{\prime}=$ $\left(B_{n}, S_{n}^{1}, \ldots, S_{n}^{\ell}, P_{n}(F)\right)$ é livre de arbitragem e que $P_{N}(F)=F$. Agora, pelo Teorema 3.22 concluímos que existe uma probabilidade neutra ao risco $\mathbb{Q}$ para $M_{n}^{\prime}$. Por ser probabilidade neutra ao risco em $M_{n}^{\prime}$, o processo $\left\{\left(\bar{S}_{n}, P_{n}(F)\left(B_{n}\right)^{-1}\right), \mathcal{F}_{n} ; n \in \overline{0, N}\right\}$ é um martingal em relação a $\mathbb{Q}$, 
com $\left\{\bar{S}_{n}, \mathcal{F}_{n} ; n \in \overline{0, N}\right\}$ sendo um martingal que toma valores em $\mathbb{R}^{\ell}$ e $\left\{P_{n}(F)\left(B_{n}\right)^{-1}, \mathcal{F}_{n} ; n \in \overline{0, N}\right\}$ um martingal em $\mathbb{R}$. Como $\left\{\bar{S}_{n}, \mathcal{F}_{n} ; n \in \overline{0, N}\right\}$ é um martingal em relação a $\mathbb{Q}$, vemos que $\mathbb{Q}$ é uma probabilidade neutra ao risco para o mercado $M_{n}=\left(B_{n}, S_{n}^{1}, \ldots, S_{n}^{\ell}\right)$. O Lema 3.25 nos leva a concluir que $\left\{\overline{V_{n}^{\Gamma}}, \mathcal{F}_{n} ; n \in \overline{0, N}\right\}$ é um martingal com valores em $\mathbb{R}$ em relação a $\mathbb{Q}$.

Como $P_{n}(F)$ ser um processo de preços corretos para $F$ e $\Gamma_{n}$ é uma cobertura de $F$, teremos

$$
P_{N}(F)\left(B_{N}\right)^{-1}=B_{N}^{-1} F=V_{N}^{\Gamma}\left(B_{N}\right)^{-1}
$$

Daí, resulta que $\left\{\overline{V_{n}^{\Gamma}}, \mathcal{F}_{n} ; n \in \overline{0, N}\right\}$ e $\left\{P_{n}(F)\left(B_{n}\right)^{-1}, \mathcal{F}_{n} ; n \in \mathbb{N}\right\}$ são martingais que assumem valores em $\mathbb{R}$ com respeito a $\mathbb{Q}$. Como $\Gamma_{n}$ é cobertura do contrato temos $\overline{V_{n}^{\Gamma}}=P_{n}(F) B_{n}^{-1} \forall n \in \overline{0, N}$ pelo Lema 3.23.

Para mostrar que $V_{n}^{\Gamma}$ é de fato o processo de preços corretos para $F$, devemos mostrar que o mercado $M_{n}^{\prime}=\left(B_{n}, \ldots S_{n}^{\ell}, V_{n}^{\Gamma}\right)$ é livre de arbitragem. Por hipótese sabemos que $M_{n}$ é mercado livre de arbitragem, logo possui probabilidade neutra ao risco $\mathbb{Q}$ e sabemos que $\left\{\bar{S}_{n}, \mathcal{F}_{n} ; n \in \overline{0, N}\right\}$ é um martingal com respeito a $\mathbb{Q}$. Também podemos inferir que $\left\{\overline{V_{n}^{\Gamma}}, \mathcal{F}_{n} ; n \in \overline{0, N}\right\}$ é um martingal com respeito a $\mathbb{Q}$ graças ao Lema 3.25. Dessa maneira, $\mathbb{Q}$ também é solução do problema martingal para $\left(\bar{S}_{n}, \overline{\left.V_{n}^{\Gamma}\right)}\right.$. Então pelo Teorema $3.22, M_{n}^{\prime}$ é livre de arbitragem.

Mostramos que $V_{n}^{\Gamma}$ tem que ser o processo de preços correto para $F$. Como $\left\{P_{n}(F)\left(B_{n}\right)^{-1}, \mathcal{F}_{n} ; n \in \overline{0, N}\right\}$ é martingal em relação a $\mathbb{Q}$, temos

$$
\mathbb{E}_{\mathbb{Q}}\left(\frac{P_{N}(F)}{B_{N}}\right)=P_{0}(F)
$$

Vamos mostrar que mesmo que o contrato $F$ não tenha cobertura, poderemos calcular seu preço correto com a expressão acima

Proposição 3.27 Seja $M_{n}$ livre de arbitragem, um contrato $F: \Omega \longrightarrow \mathbb{R}$, e $\mathbb{Q}$ uma probabilidade neutra ao risco. Então

$$
P_{n}(F)=B_{n} \mathbb{E}_{\mathbb{Q}}\left(\frac{F}{B_{N}} \mid \mathcal{F}_{n}\right)
$$

é um processo de preços corretos para $F$.

Prova. Primeiro vemos que $P_{N}(F)=F$. Agora devemos mostrar que o mercado $M_{n}^{\prime}=\left(B_{n}, S_{n}^{1}, \ldots S_{n}^{\ell}, P_{n}(F)\right)$ é livre de arbitragem.

Como $\mathbb{Q}$ é probabilidade neutra ao risco, $\left\{\bar{S}_{n}, \mathcal{F}_{n} ; \overline{0, N}\right\}$ é um martingal com relação a $\mathbb{Q}$ e $B_{n}^{-1} P_{n}(F)=\mathbb{E}_{\mathbb{Q}}\left(F \mid \mathcal{F}_{n}\right)$ também é, pelo ponto 2 do Lema 3.8. 
Agora, notemos que $\mathbb{Q}$ é solução do problema martingal de $\left(1, \bar{S}_{n}, P_{n}(F) B_{n}^{-1}\right)$. Pelo Teorema 3.22 temos que $M_{n}^{\prime}$ é livre de arbitragem.

A próxima e última proposição relaciona o que vimos sobre o processo de preços corretos de um contrato $F$ com a existência de uma medida de probabilidade neutra ao risco.

Proposição 3.28 Seja $M_{n}$ livre de arbitragem, $F: \Omega \longrightarrow \mathbb{R}$ um contrato e $P_{n}(F)$ um processo de preços correto para este contrato. Então existe $\mathbb{Q}$ probabilidade neutra ao risco tal que $P_{n}(F)=B_{n} \mathbb{E}_{\mathbb{Q}}\left(\frac{F}{B_{N}} \mid \mathcal{F}_{n}\right), \forall n \in \overline{0, N}$.

Prova. Por hipótese, sabemos que $P_{n}(F)$ é um processo de preços corretos, $\operatorname{logo} P_{N}(F)=F$ e $M_{n}^{\prime}$ é livre de arbitragem. Pelo Teorema 3.22 vemos que existe $\mathbb{Q}$ probabilidade neutra ao risco para $M_{n}^{\prime}$. Essa mesma $\mathbb{Q}$ é também probabilidade neutra ao risco $M_{n}$, pois com a exclusão da última coordenada o vetor aleatório $\left\{\left(1, \bar{S}_{n}\right), \mathcal{F}_{n} ; \overline{0, N}\right\}$ continua sendo um martingal com respeito a $\mathbb{Q}$. Agora, $\left\{B_{n}^{-1} P_{n}(F), \mathcal{F}_{n} ; \overline{0, N}\right\}$ é um martingal. Daí,

$$
\mathbb{E}_{\mathbb{Q}}\left(B_{N}^{-1} P_{N}(F) \mid \mathcal{F}_{n}\right)=B_{n}^{-1} P_{n}(F)
$$

pelo ponto 2 da Proposição 3.7. Isso prova o que desejávamos. 


\section{4}

\section{Modelo Binomial}

O modelo usado como ilustração da formalização que apresentamos é o modelo binomial com vários períodos de tempo ou modelo CRR (Cox-RossRubinstein), com um ativo livre de risco e um ativo com risco. No Capítulo 2 o principal exemplo era um modelo binomial com dois períodos de tempo. Trataremos do caso com tempo final $N \in \mathbb{N}$.

$\mathrm{O}$ ativo livre de risco varia de forma previsível entre quaisquer duas datas com uma taxa fixa $r>-1$ que mantém o preço positivo, logo $B_{n}=B_{n-1}(1+r)$ para $n \in\{\overline{1, N}\}$. O ativo arriscado tem retorno $R_{n}=\frac{S_{n}-S_{n-1}}{S_{n-1}}$ que depende do comportamento de $S_{n}$. Neste modelo assumimos que $S_{n}$ pode comportar-se de duas maneiras: valorizando com a taxa $u$ ou desvalorizando com a taxa $d$. Devemos ter $-1<d<u$. para que os preços sempre sejam positivos no modelo. A probabilidade de que o ativo se valorize é fixa em todas as datas do modelo

O ativo arriscado terá um comportamento como exemplificado pela figura 4.1 mais adiante. Este comportamento pode ser inferido para $n$ períodos de tempo. A cada nó há sempre duas formas de variação do preço do ativo, o que dá um caráter binário ao modelo. Na data final veremos que existem $2^{N}$ trajetórias possíveis para o preço $S_{n}$. Desta forma podemos definir nosso espaço amostral $\Omega$ como o conjunto de todas as sequências possíveis de tamanho $N$ formadas por coordenadas representado valorizações ou desvalorizações. Mais formalmente

$$
\Omega=\{-1,+1\}^{N}=\left\{\omega=\left(x_{1}, x_{2}, \ldots x_{N}\right) \mid x_{i} \in\{-1,+1\} \text {, para } i \in \overline{1, N}\right\}
$$

Façamos $\pi_{n}(\omega)=x_{n}$ a projeção da $n$-ésima coordenada de $\omega$. Podemos escrever o retorno do ativo arriscado como

$$
R_{n}(\omega)=\frac{u\left(1-\pi_{n}(\omega)\right)}{2}+\frac{d\left(1+\pi_{n}(\omega)\right)}{2}
$$

onde $R_{n}(\omega)=u$ quando $\pi_{n}(\omega)=-1$ e $R_{n}(\omega)=d$ quando $\pi_{n}(\omega)=1$. Agora, 
podemos escrever o processo de preços do ativo arriscado como

$$
S_{n}=S_{0} \prod_{k=1}^{n}\left(1+R_{k}\right)
$$

ou e o processo de preços descontados como

$$
\overline{S_{n}}=\frac{S_{0} \prod_{k=1}^{n}\left(1+R_{k}\right)}{(1+r)^{n}}=S_{0} \prod_{k=1}^{n} \frac{1+R_{k}}{1+r} .
$$

Escrevemos explicitamente o preço $\overline{S_{n}}$ em função do preço $\overline{S_{n-1}}$ como

$$
\overline{S_{n}}=\frac{S_{0} \prod_{k=1}^{n}\left(1+R_{k}\right)}{(1+r)^{n}}=\left(\frac{S_{0} \prod_{k=1}^{n-1}\left(1+R_{k}\right)}{(1+r)^{n-1}}\right)\left(\frac{1+R_{n}}{1+r}\right)=\overline{S_{n-1}}\left(\frac{1+R_{n}}{1+r}\right) .
$$

A $\sigma$-álgebra $\mathcal{F}$ que vamos considerar é a formada por todos os subconjuntos de $\Omega$ definido acima. Para a dinâmica da informação podemos usar os instrumentos de processos estocásticos que elaboramos (veja a Definição A.11). Definimos $\mathcal{F}_{n}=\sigma\left\{S_{1}, \ldots S_{n}\right\}=\sigma\left\{R_{1}, \ldots R_{n}\right\}$. Desta forma $\mathcal{F}_{n} \subseteq \mathcal{F}_{n+1}$ e $\mathcal{F}_{N}=\mathcal{F}$. O processo de preços $\left\{S_{n}, n \in \overline{0, N}\right\}$ é um processo estocástico em que cada $S_{n}$ é mensurável com relação à sigma-álgebra $\mathcal{F}_{n}$.

A seguinte proposição já foi demonstrada para um caso tratado no Capítulo 2, num modelo com dois períodos de tempo e na sequência do trabalho, onde vimos que aquele modelo era completo. O modelo binomial que tratamos neste capítulo é uma sequência de diversos modelos de dois períodos de tempo conectados. Intuitivamente somos levados a crer que o modelo binomial seja completo. Mostraremos tal fato.

Proposição 4.1 O modelo binomial é livre de arbitragem se e somente se $d<r<u$. O modelo é completo.

Prova. Sabemos que o modelo é livre de arbitragem se $Q_{\bar{S}} \neq \emptyset$ (veja o Teorema 3.2) e que $\left\{\bar{S}_{n}, \mathcal{F}_{n} ; n \in \overline{0, N}\right\}$ é um martingal com relação a alguma medida de probabilidade em $Q_{\bar{S}}$. Desta forma

$$
\begin{aligned}
\overline{S_{n}} & =\mathbb{E}_{\mathbb{Q}}\left(\overline{S_{n+1}} \mid \mathcal{F}_{n}\right) \text { e pela } \operatorname{Equação}(4-1), \\
& =\overline{S_{n}} \mathbb{E}_{\mathbb{Q}}\left(\frac{1+R_{n+1}}{1+r} \mid \mathcal{F}_{n}\right) .
\end{aligned}
$$

Logo $r=\mathbb{E}_{\mathbb{Q}}\left(R_{n+1} \mid \mathcal{F}_{n}\right)$. Usando a esperança e sabendo que o ativo com risco só pode se valorizar ou desvalorizar segundo uma probabilidade fixa, temos

$$
\begin{aligned}
& r=\mathbb{Q}\left(R_{n+1}=u \mid \mathcal{F}_{n}\right) \cdot u+\mathbb{Q}\left(R_{n+1}=d \mid \mathcal{F}_{n}\right) \cdot d \\
& r=\mathbb{Q}\left(R_{n+1}=u \mid \mathcal{F}_{n}\right) \cdot u+\left(1-\mathbb{Q}\left(R_{n+1}=u \mid \mathcal{F}_{n}\right)\right) \cdot d
\end{aligned}
$$


Então,

$$
p^{*}=\frac{r-d}{u-d}
$$

em que fizemos $p^{*}=\mathbb{Q}\left(R_{n+1}=u \mid \mathcal{F}_{n}\right)(\omega), \omega \in \Omega$. A expressão que explicita a probabilidade quer dizer que, dentro da sequência finita $\omega=\left(x_{1}, x_{2}, \ldots, x_{N}\right)$ formada por $-1 \mathrm{e}+1$, o termo $x_{n+1}$ tem probabilidade $p^{*}$, dada a informação disponível até o tempo $n$, de ser $x_{n+1}=-1$ e consequentemente $R_{n+1}(\omega)=u$, $\forall n \in \overline{1, N-1}$.

Note que a probabilidade $p^{*}=\mathbb{Q}\left(R_{n+1}=u \mid \mathcal{F}_{n}\right)$ não tem nenhum componente aleatório, ou seja, não depende dos valores de $R_{1}, R_{2}, \ldots, R_{n}$. Como consequência, $R_{n+1}(\omega)$ não depende da informação contida em $\mathcal{F}_{n}$, ou seja, não depende de $R_{1}, \ldots, R_{n}$. Com isso, vemos que as variáveis aleatórias $R_{n}, n \in \overline{1, N}$ são independentes e identicamente distribuídas, com distribuição dada por

$$
\begin{aligned}
& \mathbb{Q}\left(R_{n+1}=u\right)=\mathbb{Q}\left(x_{n+1}=-1\right)=p^{*}=\frac{r-d}{u-d} \\
& \mathbb{Q}\left(R_{n+1}=d\right)=\mathbb{Q}\left(x_{n+1}=+1\right)=1-p^{*} .
\end{aligned}
$$

Logo, vemos que o modelo é livre de arbitragem se e somente se $d<r<u$ como foi visto no Capítulo 2. A probabilidade neutra ao risco é única por construção e pelo Teorema 3.3 o modelo é completo.

Como $R_{1}, R_{2}, \ldots R_{N}$ são independentes e identicamente distribuídas, uma sequência $\omega \in \Omega$ com $k$ termos iguais a -1 e $N-k$ termos iguais a +1 terá probabilidade dada por

$$
\mathbb{Q}(\omega)=p^{* k}\left(1-p^{*}\right)^{N-k}
$$

O modelo binomial fica então definido no espaço filtrado $(\Omega, \mathcal{F}, \mathbb{Q}),\left\{\mathcal{F}_{n} ; n \in \overline{0, N}\right\}$.

Note que quando desejarmos saber a probabilidade do preço do ativo com risco ser $S_{0}(1+u)^{k}(1+d)^{N-k}$, deveremos considerar todas as possíveis trajetórias até obtermos este preço. Para isso, calculamos

$$
\mathbb{Q}\left(S_{N}=S_{0}(1+u)^{k}(1+d)^{N-k}\right)=\left(\begin{array}{c}
N \\
k
\end{array}\right) p^{* k}\left(1-p^{*}\right)^{N-k} .
$$

Ao longo do trabalho os contratos foram tratados de forma muito genérica 
como uma variável aleatória que assume valores positivos. Na prática existem diversos tipos de contratos, cada um com regras que definem como e quando será feito o pagamento ao possuidor (payoff) no período de vencimento, sendo que este também pode ser variável. O tipo mais simples de contrato tratado são as opções européias. As opções européias são um tipo de contrato em que o pagamento de payoff no período de expiração $N$ depende do preço do ativo subjacente $S_{N}$. Existem as opções que dão ao comprador o direito de adquirir o ativo arriscado a um preço previamente combinado $K$ (chamadas de call européia) ou então de vender (put européia). Ao emitir um contrato no estilo opção européia, fica combinado um valor $K$ chamado strike e o comprador tem a escolha de na data de expiração $N$ poder comprar um ativo $S$ ao preço $K$ ou vendê-lo, segundo o tipo de contrato que escolheu.

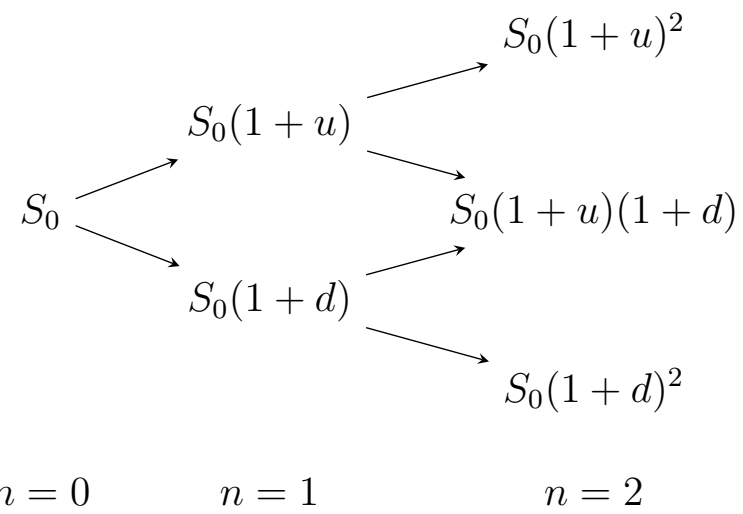

Figura 4.1: Árvore Binomial de dois intervalos temporais

Desta maneira, em $N$ o payoff de uma call européia deve ser

$$
F\left(S_{N}\right)=\max \left\{S_{N}-K, 0\right\}=\left(S_{N}-K\right)^{+},
$$

ou seja, o agente deseja lucrar e para isso exercerá seu direito de comprar $S$ se em $N$ acontecer $K<S_{N}$, pois assim poderá comprar ao preço $K$ e vender a $S_{N}$ no mercado, lucrando a diferença $S_{N}-K$. O payoff de uma put européia é

$$
F\left(S_{N}\right)=\max \left\{K-S_{N}, 0\right\}=\left(K-S_{N}\right)^{+},
$$

o agente pode realizar uma operação em que pega o quantia de $S_{N}$ emprestada, adquire o ativo de risco, vende a $K$ e devolve a quantia que tomou antes, lucrando $K-S_{N}$.

Tendo apresentado este tipo de contrato, podemos utilizar o modelo binomial para precificá-lo. 
Podemos representar graficamente o modelo binomial como na Figura 4.1. Como de um período de tempo para outro o preço do ativo com risco pode se valorizar ou se desvalorizar, ocorre que a cada período de tempo as possíveis trajetórias que o preço pode seguir duplicam. O que se origina é uma figura em formato de árvore.

Para precificar uma call européia, vamos seguir um processo retroativo na estrutura em forma de árvore, de forma a encontrar os preços do contrato em cada nó do período de tempo anterior. Se a árvore só tem uma data, fazemos somente $\mathbb{E}_{\mathbb{Q}}(F)=\mathbb{E}_{\mathbb{Q}}\left(p^{*} F\left(S_{1}^{u}\right)+\left(1-p^{*}\right) F\left(S_{1}^{d}\right)\right)=P_{0}$ para encontrar o preço correto da call, aqui representada por $F$, que é uma função do preço do ativo arriscado em $n=1$. Para duas datas, devemos realizar o cálculo do valor esperado pela probabilidade neutra ao risco a partir do período $n=2$, onde a call pode assumir três valores distintos graças aos possíveis preços do ativo com risco: $F\left(S_{2}^{u u}\right), F\left(S_{2}^{d d}\right)$ ou $F\left(S_{2}^{u d}\right)=F\left(S_{2}^{d u}\right)$. São somente três valores que a call pode assumir, pois dois dos quatro possíveis preços do ativo arriscado são iguais

$$
S_{2}^{u d}=S_{0}(1+u)(1+d)=S_{0}(1+d)(1+u)=S_{2}^{d u} .
$$

Desta forma encontraremos dois valores em $n=1, P_{1}^{u}$ e $P_{1}^{d}$, que são os preços da call para cada um dos cenários de $n=1$. De $n=1$ para $n=0$ aplicaremos novamente o valor esperado pela probabilidade neutra ao risco usando os valores encontrados na primeira etapa de cálculos que realizamos (de $n=2$ para $n=1$ ). As equações que representam o que explicamos acima são as seguintes, que mostram as fórmulas para $P_{1}^{u}$ e $P_{1}^{d}$ e posteriormente para $P_{0}$ :

$$
\begin{aligned}
& P_{1}^{u}=\frac{1}{1+r} \mathbb{E}_{\mathbb{Q}}\left(p^{*} F\left(S_{2}^{u u}\right)+\left(1-p^{*}\right) F\left(S_{2}^{u d}\right)\right) \\
& P_{1}^{d}=\frac{1}{1+r} \mathbb{E}_{\mathbb{Q}}\left(p^{*} F\left(S_{2}^{d u}\right)+\left(1-p^{*}\right) F\left(S_{2}^{d d}\right)\right) .
\end{aligned}
$$

Como $S_{2}^{u d}=S_{2}^{d u}$ :

$$
P_{0}=\frac{1}{(1+r)^{2}}\left(p^{* 2} F\left(S_{2}^{u u}\right)+2 p^{*}\left(1-p^{*}\right) F\left(S_{2}^{u d}\right)+\left(1-p^{*}\right)^{2} F\left(S_{2}^{d d}\right)\right) .
$$

Para $N$ datas, podemos proceder seguindo o mesmo método, notando que sempre que as quantidades de valorizações e desvalorizações do ativo com risco são iguais então ele terá um preço igual, consequentemente a call pagará o mesmo payoff. Procedendo assim obteremos a fórmula fechada, que é exatamente $\mathbb{E}_{\mathbb{Q}}\left(\frac{F}{(1+r)^{N}}\right)=P_{0}$, citada na Proposição 3.26: 


$$
P_{0}=\frac{1}{(1+r)^{N}} \sum_{k=0}^{N}\left(\begin{array}{l}
N \\
k
\end{array}\right) p^{* k}\left(1-p^{*}\right)^{N-k} F\left(S_{0}(1+u)^{k}(1+d)^{N-k}\right) .
$$

Para encontrar o preço de uma call européia em algum período $n \in \overline{0, N}$, usamos a Proposição 3.26 e temos

$$
P_{n}(F)=\frac{1}{(1+r)^{N-n}} \sum_{k=0}^{N-n}\left(\begin{array}{c}
N-n \\
k
\end{array}\right) p^{* k}\left(1-p^{*}\right)^{N-n-k} F\left(S_{n}(1+u)^{k}(1+d)^{N-n-k}\right)
$$

O contrato $F$ possui cobertura e pela Proposição 3.26 tem um único processo de preços corretos $P_{n}(F)=V_{n}^{\Gamma}$.

Podemos encontrar a cobertura do contrato usando uma maneira muito semelhante a que usamos do Capítulo 2, quando lidamos com um contrato no modelo com dois períodos de tempo.

Seja $\Gamma_{n}=\left(\alpha_{n}, \beta_{n}\right)$ uma estratégia auto-financiada. Seja $P_{n}(F)=V_{n-1}^{\Gamma^{u}}=$ $\Gamma_{n} \cdot\left(B_{n}, S_{n-1}(1+u)\right)$ e $P_{n}(F)=V_{n-1}^{\Gamma}{ }^{d}=\Gamma_{n} \bullet\left(B_{n}, S_{n-1}(1+d)\right)$ os dois possíveis valores do processo de preços no período $n$, que dependem da valorização ou desvalorização do ativo com risco. Logo, podemos escrever que tal estratégia gerará o valor do contrato caso haja uma valorização ou uma desvalorização do ativo com risco:

$$
\begin{aligned}
& \beta_{n} B_{n-1}(1+r)+\alpha_{n} S_{n-1}(1+u)=V_{n-1}^{\Gamma}(1+u) \\
& \beta_{n} B_{n-1}(1+r)+\alpha_{n} S_{n-1}(1+d)=V_{n-1}^{\Gamma}(1+d) .
\end{aligned}
$$

Solucionando o sistema acima, obtemos

$$
\begin{aligned}
& \alpha_{n}=\frac{V_{n-1}^{\Gamma}{ }^{u}-V_{n-1}^{\Gamma}{ }^{d}}{(u-d) S_{n-1}} \\
& \beta_{n}=\frac{1}{(1+r)}\left(\frac{(1+u) V_{n-1}^{\Gamma-1}-(1+d) V_{n-1}^{\Gamma}{ }^{u}}{u-d}\right)
\end{aligned}
$$

que é a estratégia replicadora do contrato $F$.

Mostramos então como se dá a dinâmica no modelo binomial e usando o que desenvolvemos no trabalho, concluímos algumas propriedades do modelo, como ausência de arbitragem e completude. Usando o que estudamos, vimos uma forma de encontrar o preço correto de uma call européia e sua cobertura. 


\section{5}

\section{Conclusão}

Esta dissertação consiste em um estudo introdutório à precificação de contratos em um ambiente de mercado com horizonte finito e finitos estados da natureza. Mostramos como se faz a precificação de um contrato em um ambiente simples, com dois períodos de tempo e dois estados possíveis da natureza. Expandimos o contexto para o caso em que se tem vários períodos de tempo e vários estados da natureza, sempre mostrando a importância da hipótese de não arbitragem para que a construção teórica faça sentido.

É claro que no mundo real é difícil que esta hipótese de não arbitragem se sustente a todo instante, mas há a crença de que as oportunidades de arbitragem tendem a desaparecer no mercado.

Após mostrarmos como os preços corretos de contratos se devem comportar, introduzimos as ferramentas dos processos estocásticos e as probabilidades neutras ao risco, relacionando os dois conceitos na precificação de ativos. Com isso, expandimos a análise do modelo de mercado e mostramos em que condições valem a ausência de arbitragem e a completude do mercado. Apresentamos o método da precificação de contratos usando o processo de preços corretos, que num mercado completo deve ser o valor do portfólio de cobertura. Por fim, ainda apresentamos um exemplo que ilustra o desenvolvimento teórico do trabalho. No modelo binomial pudemos mostrar algumas aplicações da teoria de precificação que foi elaborada durante o trabalho. Nele, conseguimos uma fórmula explícita para o preço correto de uma opção de compra européia. Mostramos a completude e a ausência de arbitragem do modelo.

A intuição adquirida aqui com o estudo dos modelos a tempo discreto pode ser expandida para modelos a tempo contínuo com as devidas adaptações. Isto é trabalho para o futuro. 


\section{Referências bibliográficas}

[1] BERTSIMAS, DIMITRIS AND TSITSIKLIS, JOHN N. Introduction to linear optimization. Athena Scientific Belmont, MA, 1997.

[2] BINGHAM, NICHOLAS H AND KIESEL, RÜDIGER. Risk-neutral valuation: Pricing and hedging of financial derivatives. Springer Science \& Business Media, 2013.

[3] PLISKA, STANLEY. Introduction to mathematical finance. Blackwell publishers Oxford UK, 1997.

[4] DURRETT, RICK. Probability: theory and examples. Cambridge university press, 2010.

[5] CAPINSKI, MAREK AND ZASTAWNIAK, TOMASZ. Mathematics for finance: an introduction to financial engineering. Springer, 2006.

[6] FÖLLMER, HANS AND SCHIED, ALEXANDER. Stochastic finance: an introduction in discrete time. Walter de Gruyter, 2011.

[8] RAMÍREZ, JOHEL. Modelo Finito de Mercados: opciones europeas y americanas. Departamento de Ciencias/Sección Matemáticas, PUCP, 2007.

[9] SHREVE, STEVEN. Stochastic calculus for finance I: the binomial asset pricing model. Springer Science \& Business Media, 2012.

[10] MEDINA, PABLO KOCH AND MERINO, SANDRO. Mathematical Finance and Probability. Springer, 2003.

[11] WILLIAMS, RUTH J. Introduction to the Mathematics of Finance, volumen 72. American Mathematical Society, 2006.

[12] BLACK, FISCHER AND SCHOLES, MYRON. The pricing of options and corporate liabilities. Journal of political economy, 81(3):637-654, 1973.

[13] COX, JOHN C AND ROSS, STEPHEN A AND RUBINSTEIN, MARK. Option pricing: A simplified approach. Journal of financial Economics, $7(3): 229-263,1979$. 


\section{Probabilidade}

Este Apêndice trata de conceitos da Teoria de Probabilidade importantes para a compreensão do texto. Muitos resultados são bem conhecidos na teoria, portanto algumas provas serão omitidas. Nem todos os resultados serão explicitamente usados no texto, porém tem importância dentro da teoria e em demonstrações de outros resultados.

\section{A.1}

\section{Espaços Mensuráveis e Funções Mensuráveis}

Os conceitos e definições apresentados aqui fazem parte da construção formal da teoria da probabilidade. Vamos nos restringir ao caso de tempo discreto e espaço amostral finito.

Considere um conjunto finito $\Omega$ e uma coleção $\mathcal{F}$ de subconjuntos deste conjunto. Podemos classificar essa coleção como sendo uma $\sigma$-álgebra segundo algumas propriedades que $\mathcal{F}$ possua.

Definição A.1 $\mathcal{F}$ é uma $\sigma$-álgebra se é munido das seguintes propriedades:

$-\Omega \in \mathcal{F}$

- Se $X \in \mathcal{F}$ então $\Omega \backslash X \in \mathcal{F}$

- Se $\left\{A_{i} ; i \in \mathbb{N}\right\}$ é uma sequência enumerável formada de conjuntos $A_{i}$ tal que $A_{i} \in \mathcal{F}$, então $\cup_{i \in \mathbb{N}} A_{i} \in \mathcal{F}$.

A partir da definição dada acima é possível verificar alguns fatos imediatos. Se $\mathcal{F}$ é $\sigma$-álgebra, é claro que $\emptyset \in \mathcal{F}$. Também, toda união finita de conjuntos de $\mathcal{F}$ está em $\mathcal{F}$, basta em uma sequência infinita enumerável de conjuntos $B_{i} \in \mathcal{F}$ fazer $B_{i}=B_{k} \forall i \geqslant k, \operatorname{logo} \cup_{i \in \mathbb{N}}^{k} B_{i} \in \mathcal{F}$. Como a união de uma sequência enumerável está em $\mathcal{F}$, tomando o complemento teremos que a interseção também estará em $\mathcal{F}$. Então a $\sigma$-álgebra é fechada para uniões e interseções enumeráveis. Esta última propriedade é a que basicamente distingue uma $\sigma$ álgebra de uma álgebra, já que uma álgebra é fechada apenas para uniões finitas.

A menor $\sigma$-álgebra de subconjuntos de $\Omega$ é o conjunto $\{\emptyset, \Omega\}$, chamada de $\sigma$-álgebra trivial e denotada por $\mathcal{T}$. A maior $\sigma$-álgebra é o chamado conjunto 
das partes do conjunto $\Omega$, denotado por $\mathcal{P}(\Omega)$, que inclui todos os subconjuntos possíveis de $\Omega$ e suas relações de união e de complementaridade. Para o caso de $\Omega$ ser finito ou enumerável, sabemos que a cardinalidade do conjunto $\mathcal{P}(\Omega)$ será $2^{|\Omega|}$.

Um caso importante é o dos números reais. De modo geral, em $d$ dimensões, definimos a coleção de subconjuntos $\mathcal{B}\left(\mathbb{R}^{d}\right)$ como a menor $\sigma$-álgebra gerada por conjuntos abertos de $\mathbb{R}^{d}$. Ou seja, considere $\mathcal{S}$ como a família dos subconjuntos abertos de $\mathbb{R}^{d}$, estamos afirmando que $\mathcal{B}\left(\mathbb{R}^{d}\right)=\sigma(S)$. A menor $\sigma$-álgebra formada pelos abertos de $\mathbb{R}^{d}$ é a chamada $\sigma$-álgebra dos borelianos de $\mathbb{R}^{d}$.

Definição A.2 Quando um conjunto $\Omega$ é munido de uma $\sigma$-álgebra $\mathcal{F}$, dizemos que o par $(\Omega, \mathcal{F})$ é um espaço mensurável.

Definição A.3 Dados dois espaços mensuráveis $\left(\Omega_{1}, \mathcal{F}_{1}\right)$ e $\left(\Omega_{2}, \mathcal{F}_{2}\right)$, dizemos que uma função $H: \Omega_{1} \longrightarrow \Omega_{2}$ é mensurável se para todo conjunto $B \in \mathcal{F}_{2}$ temos $H^{-1}(B) \in \mathcal{F}_{1}$.

O caso de função mensurável usado neste trabalho é o de uma variável aleatória.

Definição A.4 Uma função $H: \Omega \longrightarrow \mathbb{R}$ é dita uma variável aleatória quando dados os espaços mensuráveis $(\Omega, \mathcal{F})$ e $(\mathbb{R}, \mathcal{B}(\mathbb{R}))$, $H$ é uma função mensurável. Dizemos que $H$ é um vetor aleatório quando é mensurável com respeito a $(\Omega, \mathcal{F})$ $e\left(\mathbb{R}^{d}, \mathcal{B}\left(\mathbb{R}^{d}\right)\right)$.

Uma função mensurável pode gerar uma $\sigma$-álgebra em seu domínio usando pré-imagens de conjuntos de sua imagem. Formalmente

Definição A.5 Sejam $\left(\Omega_{1}, \mathcal{F}_{1}\right)$ e $\left(\Omega_{2}, \mathcal{F}_{2}\right)$ espaços mensuráveis fixados. Seja a função $H: \Omega_{1} \longrightarrow \Omega_{2}$ uma função mensurável. Então o conjunto $\mathcal{F}_{H}=$ $\left\{H^{-1}(B) \subseteq \Omega_{1} ; B \in \mathcal{F}_{2}\right\}$ é uma $\sigma$-álgebra e diz-se a $\sigma$-álgebra gerada por $H$.

Definição A.6 Considere os espaços mensuráveis $\left(\Omega_{1}, \mathcal{F}_{1}\right)$ e $\left(\Omega_{2}, \mathcal{F}_{2}\right)$. Seja uma função mensurável $H: \Omega_{1} \longrightarrow \Omega_{2}$. Seja uma $\sigma$-álgebra $\mathcal{G} \subseteq \mathcal{F}_{1}$. Dizemos que a variável $H$ é $\mathcal{G}$-mensurável se $\mathcal{F}_{H} \subseteq \mathcal{G}$.

Definição A.7 Diremos que $\pi=\left\{A_{1}, A_{2}, \ldots, A_{k}\right\}$ é uma partição do conjunto $\Omega$ se $\Omega=\cup_{i=1}^{k} A_{i}$ e $A_{i} \cap A_{j}=\emptyset \forall i \neq j$.

Proposição A.8 Se $\mathcal{G}$ é uma $\sigma$-álgebra finita, ou seja, uma $\sigma$-álgebra com cardinalidade finita, então existe uma partição $\pi$ que gera $\mathcal{G}$, ou seja $\mathcal{G}=\mathcal{G}(\pi)$, representando a $\sigma$-álgebra gerada pela partição. 
Proposição A.9 Seja $(\Omega, \mathcal{F})$ um espaço mensurável e $\mathcal{F}$ uma $\sigma$-álgebra finita. Denotemos por $\pi=\left\{A_{1}, A_{2}, \ldots A_{k}\right\}$ a partição de $\Omega$ que gera $\mathcal{F}$. A variável aleatória $H: \Omega \longrightarrow \mathbb{R}^{d}$ é constante sobre cada conjunto $A_{j}$.

Prova. Tomemos um elemento arbitrário $A_{j}$ da partição $\pi$ e vamos supor que a função $H$ não seja constante sobre $A_{j}$. Se só existe um elemento em $A_{j}$ então isto é um absurdo. Suponhamos então que $A_{j}=\left\{a_{1}, a_{2}, \ldots a_{n}\right\}$ e $H\left(A_{j}\right)=\left\{c_{1}, c_{2}, \ldots c_{\ell}\right\}$ com $c_{i} \neq c_{j} \forall i \neq j$, ou seja, $H$ não é constante em $A_{j}$ e assume $\ell$ valores distintos. Seja $B_{1}=\left\{c_{1}\right\}, \ldots, B_{\ell}=\left\{c_{\ell}\right\}$ e assim $H\left(A_{j}\right)=B_{1} \cup \ldots \cup B_{\ell}$. É claro que $A_{j} \subseteq H^{-1}\left(B_{1}\right) \cup \ldots \cup H^{-1}\left(B_{\ell}\right)$. Como $\forall i \in\{1,2, \ldots \ell\}, \quad H^{-1}\left(B_{i}\right) \in \mathcal{F}$ então cada $H^{-1}\left(B_{i}\right)$ é gerado por elementos da partição $\pi$. Dessa forma, vemos que $A_{i}$ deve estar contido em uma união de elementos de $\mathcal{F}$ gerados por $\pi$, logo não poderia ser um elemento da partição $\pi$, um absurdo.

\section{A. 2}

\section{Processos Estocásticos}

Aqui apresentamos as ferramentas necessárias para a modelagem do mercado num ambiente em que novas informações são agregadas a cada instante de tempo $n$. Consideraremos o tempo discreto, ou seja, $n \in \mathbb{N}$.

Definição A.10 Seja $\Omega$ um conjunto finito e fixo. Uma sequência de $\sigma$ álgebras $\left\{\mathcal{F}_{n} ; n \in \mathbb{N}\right\}$ é chamada filtro, se

$$
\mathcal{F}_{n} \subseteq \mathcal{F}_{n+1} \ldots \forall n \in \mathbb{N}
$$

Neste caso diz-se que é uma sequência crescente de $\sigma$-álgebras.

Um espaço mensurável $(\Omega, \mathcal{F})$ é dito filtrado quando fixamos um filtro $\left\{\mathcal{F}_{n} ; n \in \mathbb{N}\right\}$, onde para cada $n \in \mathbb{N}$ temos $\mathcal{F}_{n} \subseteq \mathcal{F}$. Com isso podemos dar a definição formal de um processo estocástico.

Definição A.11 Um processo estocástico que assume valores em $\mathbb{R}^{d}$ consiste num espaço filtrado e uma sequência de vetores aleatórios $\left\{X_{n} ; n \in \mathbb{N}\right\}$, $X_{n}:(\Omega, \mathcal{F}) \longrightarrow\left(\mathbb{R}^{d}, \mathcal{B}\left(\mathbb{R}^{d}\right)\right)$ tal que cada $X_{n}$ é $\mathcal{F}_{n}$-mensurável.

Se $X_{n}$ é um processo estocástico assumindo valores em $\mathbb{R}^{d}$, então $X_{n}=$ $\left(X_{n}^{1}, X_{n}^{2}, \ldots, X_{n}^{d}\right)$ onde cada coordenada é um processo estocástico que toma valores em $\mathbb{R}$, i.e, $X_{n}^{i}:(\Omega, \mathcal{F}) \longrightarrow(\mathbb{R}, \mathcal{B}(\mathbb{R}))$ é um processo estocástico $\forall i \in\{1,2, \ldots d\}$.

Os portfólios serão modelados usando os processos previsíveis. 
Definição A.12 Fixado o espaço filtrado $(\Omega, \mathcal{F}),\left\{\mathcal{F}_{n} ; n \in \mathbb{N}\right\}$, dizemos que um processo $\left\{H_{n} ; n \in \mathbb{N}\right\}$ que toma valores em $\mathbb{R}^{d}$ é previsivel se $\forall n \in \mathbb{N}$, $H_{n+1}$ é $\mathcal{F}_{n}$-mensurável.

Apresentamos agora uma operação importante também conhecida por transformação martingal.

Definição A.13 Sejam $X_{n}$ e $Y_{n}$ dois processos estocásticos assumindo valores em $\mathbb{R}^{d}$ definidos sob o mesmo espaço filtrado $(\Omega, \mathcal{F}),\left\{\mathcal{F}_{n} ; n \in \mathbb{N}\right\}$, com $Y_{n}$ previsivel. Com isso, definimos o novo processo $Z_{n}$, onde $Z_{0}=0$ como

$$
Z_{n}=\sum_{i=1}^{n} Y_{i} \cdot\left(X_{i}-X_{i-1}\right), \quad \forall n \geqslant 1
$$

que denotamos por:

$$
Z_{n}=\int_{0}^{n} Y d X
$$

Usaremos também a notação

$$
Z_{m}-Z_{n}=\int_{n}^{m} Y d X, \quad \forall m, n \geqslant 0
$$

para representarmos o processo acima.

As definições apresentadas irão ajudar na modelagem da dinâmica de informação do mercado. O filtro representará o acréscimo de informação que ocorre a cada instante de tempo que passa e com isso mais se conhece a respeito do comportamento das variáveis aleatórias envolvidas na modelagem.

\section{A.3}

\section{Independência e Esperança Condicional}

Considere o espaço mensurável $(\Omega, \mathcal{F})$. Seja $\mathbb{P}: \mathcal{F} \longrightarrow[0,1]$ uma medida de probabilidade. Se adicionarmos a medida de probabilidade ao espaço mensurável anterior, diremos que o espaço $(\Omega, \mathcal{F}, \mathbb{P})$ é um espaço de probabilidade.

Definição A.14 Seja $\left(\Omega_{1}, \mathcal{F}_{1}\right)$ um espaço mensurável e seja $H:(\Omega, \mathcal{F}) \longrightarrow$ $\left(\Omega_{1}, \mathcal{F}_{1}\right)$ uma função mensurável. Definiremos $\mu_{H}$ como a medida induzida por $H$ sobre $\left(\Omega_{1}, \mathcal{F}_{1}\right)$, da seguinte forma:

$$
\mu_{H}(B)=\mathbb{P}\left(H^{-1}(B)\right), \quad \forall B \in \mathcal{F}_{1}
$$

Como para $B \in \mathcal{F}_{1}, H^{-1}(B) \in \mathcal{F}$ temos $\mu_{H}$ bem definida. Chamamos a $\mu_{H}$ a distribuição de $H$. 
No espaço de probabilidade $(\Omega, \mathcal{F}, \mathbb{P})$ consideraremos nossos conjuntos. Sejam $\mathcal{G}_{1}, \mathcal{G}_{2}, \ldots \mathcal{G}_{k}$ sub $\sigma$-álgebras de $\mathcal{F}$. Vamos definir independência entre $\sigma$-álgebras e entre variáveis aleatórias para depois tratarmos da esperança condicional.

Definição A.15 As $\sigma$-álgebras $\mathcal{G}_{1}, \mathcal{G}_{2}, \ldots \mathcal{G}_{k}$ são ditas independentes se $\forall A_{i} \in$ $\mathcal{G}_{i}$ e $i \in\{1,2, \ldots, k\}$, tivermos

$$
\mathbb{P}\left(\cap_{i=1}^{k} A_{i}\right)=\prod_{i=1}^{k} \mathbb{P}\left(A_{i}\right)
$$

Definição A.16 Sejam $X_{1}, X_{2}, \ldots X_{k}$ variáveis aleatórias definidas no espaço de probabilidade $(\Omega, \mathcal{F}, \mathbb{P})$. As variáveis são ditas independentes se as $\sigma$ álgebras geradas pelas variáveis aleatórias são independentes, i.e, as $\sigma$-álgebras $\mathcal{F}_{X_{1}}, \mathcal{F}_{X_{2}}, \ldots, \mathcal{F}_{X_{k}}$ são independentes.

O conceito de esperança e o conceito de integrabilidade são muito importantes em definições posteriores. Cabe agora definirmos o que são.

Definição A.17 Seja $f:(\Omega, \mathbb{F}) \longrightarrow(\mathbb{R}, \mathcal{B}(\mathbb{R}))$ uma função mensurável tal que $f \in \mathcal{L}^{p}(\Omega, \mathcal{F}, \mathbb{P})$, ou seja, $f$ faz parte da classe das funções $p$-integráveis, com $p \in \mathbb{R}$ e $p \geqslant 1$. Dizemos que $f$ é $p$-integrável se $\int|f|^{p} d \mathbb{P}<\infty$. Para o caso em que $p=1$ dizemos somente que $f$ é integrável. Note que $f$ é integrável se e somente se $|f|$ é integrável.

Mais detalhes acerca da definição anterior podem ser encontrados em [4] ou em alguma obra de Teoria da Medida e Integração, bem como em obras de Teoria da Probabilidade.

Definição A.18 Considere uma variável aleatória $X$ tal que $X \in \mathcal{L}^{1}(\Omega, \mathcal{F}, \mathbb{P})$. Definimos a esperança de $X$ por

$$
\mathbb{E}(X):=\int_{\Omega} X d \mathbb{P}
$$

Quando em um espaço de probabilidade como o definido anteriormente desejamos conhecer a esperança de uma variável aleatória havendo restrição de informação disponível, estamos tratando do conceito de esperança condicional. Por exemplo, consideremos a variável aleatória $X:(\Omega, \mathcal{F}) \longrightarrow(\mathbb{R}, \mathcal{B}(\mathbb{R}))$ e uma sub $\sigma$-álgebra $\mathcal{G} \subset \mathcal{F}$. A esperança condicional $\mathbb{E}(X \mid \mathcal{G})$ é uma variável aleatória que melhor aproxima o valor de $X$ quando restrita a $\mathcal{G}$. 
Definição A.19 Seja $X: \Omega \longrightarrow \mathbb{R}$ uma variável aleatória integrável. Definimos $\mathbb{E}(X \mid \mathcal{G})$ como uma variável aleatória $Z:(\Omega, \mathcal{F}) \longrightarrow(\mathbb{R}, \mathcal{B}(\mathbb{R}))$ tal que

1. $Z$ é $\mathcal{G}$-mensurável;

2. $\forall A \in \mathcal{G}$ temos

$$
\int_{A} X d \mathbb{P}=\int_{A} Z d \mathbb{P}
$$

Qualquer variável que cumpra as condições citadas acima é dita uma versão da esperança condicional. A existência e a unicidade em quase todo ponto da variável aleatória $Z$ é mostrada pelo Teorema de Radon-Nykodin. A prova para este fato é curta e pode ser vista em [4].

Proposição A.20 As principais propriedades da esperança condicional estão listadas abaixo:

1. $\mathbb{E}(X \mid \mathcal{T})=\mathbb{E}(X)$, lembrando que $\mathcal{T}$ é a $\sigma$-álgebra trivial;

2. Se $X$ é $\mathcal{G}$-mensurável então $\mathbb{E}(X \mid \mathcal{G})=X$;

3. Se $\mathcal{G}$ e $X$ são independentes, ou seja, $\mathcal{G}$ e $\sigma(X)$ são independentes, então $\mathbb{E}(X \mid \mathcal{G})=\mathbb{E}(X)$

4. $\mathbb{E}(X+Y \mid \mathcal{G})=\mathbb{E}(X \mid \mathcal{G})+\mathbb{E}(Y \mid \mathcal{G})$;

5. $\forall c \in \mathbb{R}, \mathbb{E}(c X \mid \mathcal{G})=c \mathbb{E}(X \mid \mathcal{G}) ;$

6. Se $Y$ é $\mathcal{G}$-mensurável, então $\mathbb{E}(X Y \mid \mathcal{G})=Y \mathbb{E}(X \mid \mathcal{G})$;

7. Se $\mathcal{G}_{2} \subset \mathcal{G}_{1}$ então $\mathbb{E}\left(\mathbb{E}\left(X \mid \mathcal{G}_{2}\right) \mid \mathcal{G}_{1}\right)=\mathbb{E}\left(X \mid \mathcal{G}_{2}\right)$;

8. Se $X \leqslant Y$ então $\mathbb{E}(X \mid \mathcal{G}) \leqslant \mathbb{E}(X \mid \mathcal{G})$;

9. $\mathbb{E}(\mathbb{E}(X \mid \mathcal{G}))=\mathbb{E}(X)$;

10. Se $\varphi: \mathbb{R} \longrightarrow \mathbb{R}$ é uma função convexa, vale a desigualdade de Jensen: $\varphi(\mathbb{E}(X \mid \mathcal{G})) \leqslant \mathbb{E}(\varphi(X) \mid \mathcal{G})$.

As demonstrações para todos os itens listados acima estão em (4).

Proposição A.21 Seja $(\Omega, \mathcal{F}, \mathbb{P})$ um espaço de probabilidade e $X: \Omega \longrightarrow \mathbb{R}$ uma variável aleatória integrável. Seja $\mathcal{G} \subset \mathcal{F}$ uma $\sigma$-álgebra gerada pela partição $\pi=\left\{A_{1}, A_{2}, \ldots A_{k}\right\}$. Então $\mathbb{E}(X \mid \mathcal{G})$ é a variável aleatória $Z: \Omega \longrightarrow \mathbb{R}$ definida por

$$
Z(\omega)=\left\{\begin{array}{c}
x_{1} \text { se } \omega \in A_{1} \\
x_{2} \text { se } \omega \in A_{2} \\
\vdots \\
x_{k} \text { se } \omega \in A_{k}
\end{array}\right.
$$


onde $x_{i}=\frac{1}{\mathbb{P}\left(A_{i}\right)} \int_{A_{i}} X d \mathbb{P}$ e $\mathbb{P}\left(A_{i}\right)>0$. Note que $x_{i}$ pode ser qualquer valor se $\mathbb{P}\left(A_{i}\right)=0$.

Prova. Pela Definição A.19 existe uma variável aleatória $Z$ tal que $\forall A_{i} \in \mathcal{G}$ vale

$$
\int_{A_{i}} X d \mathbb{P}=\int_{A_{i}} Z d \mathbb{P}=\int_{A_{i}} \mathbb{E}(X \mid \mathcal{G}) d \mathbb{P},
$$

e $Z$ é $\mathcal{G}$-mensurável. Com $X$ sendo constante sobre cada conjunto da partição (vimos isto na Proposição A.9), teremos

$$
\begin{aligned}
\int_{A_{i}} X d \mathbb{P} & =\int_{A_{i}} Z d \mathbb{P}=\int_{A_{i}} x_{i} d \mathbb{P} \\
\int_{A_{i}} X d \mathbb{P} & =x_{i} \mathbb{P}\left(A_{i}\right) \\
x_{i} & =\frac{1}{\mathbb{P}\left(A_{i}\right)} \int_{A_{i}} X d \mathbb{P}
\end{aligned}
$$

e o resultado fica provado. 